\title{
THE CONTINUITY EQUATION WITH CUSP SINGULARITIES
}

\author{
YAN LI
}

\begin{abstract}
In this paper we study a special case of the completion of cusp Kähler-Einstein metric on the regular part of varieties by taking the continuity method proposed by La Nave and Tian. The differential geometric and algebro-geometric properties of the noncollapsing limit in the continuity method with cusp singularities will be investigated.
\end{abstract}

\section{INTRODUCTION}

The Yau-Tian-Donaldson conjecture for Fano manifolds has revealed deep connections among complex Monge-Ampère equation, metric geometry and complex algebraic geometry. Some specialists develop many techniques to deal with the celebrated conjecture, see [22] or [6], [7], [8]. These methods also play an important role in studying many other problems. For instance, in [21], J. Song proves that the metric completion of the regular set of Calabi-Yau varieties and canonical models of general type with crepant singularities is a compact length space which homeomorphic to the original variety. In [18], G. La Nave and G. Tian introduce a new continuity equation to consider the analytic minimal model program. Later in [19, G. La Nave, G. Tian and Z. L. Zhang study the differential geometric and algebro-geometric properties of the noncollapsing limit in the continuity equation. These fundamental results focus on the compact Kähler manifolds. For noncompact case, let us recall some facts. Suppose $\bar{M}$ is a compact complex manifold, $D$ is an effective divisor with only normal crossings and $K_{\bar{M}}+D$ is ample, where $K_{\bar{M}}$ is the canonical line bundle over $\bar{M}$. A well known result achieved by Kobayashi [16] and Tian-Yau [23] asserts that there exists a complete negative Kähler-Einstein metric on $\bar{M} \backslash D$. Recently, in [2], two authors generalize this result. For the convenience, this consequence is stated as following (Theorem C [2]).

Theorem 1.1. Let $\bar{M}$ be a compact Kähler manifold and $D$ is a simple normal crossings $\mathbb{R}$-divisor on $\bar{M}$ with coefficients in $[-1,+\infty)$ such that $K_{\bar{M}}+D$ is semi-positive and big. Then there exists a unique $\omega$ in $c_{1}\left(K_{\bar{M}}+D\right)$ which is smooth on a Zariski open set $U$ of $\bar{M}$ and such that

$$
\operatorname{Ric}(\omega)=-\omega+[D] .
$$

More precisely, $U$ can be taken to the $\bar{M} \backslash(D \cup \mathcal{S})$, where $\mathcal{S}$ is the intersetion of all effective $\mathbb{Q}$-divisors E such that $K_{\bar{M}}+D-E$ is ample.

Motivated by [21], a natural problem is to ask what the completion of $(U, \omega)$ is. In this article, a special case is investigated. More precisely, suppose $\bar{M}$ is a projective manifold, $D$ is a smooth hypersurface and $K_{\bar{M}}+D$ is big and semi-ample. According to the Kawamata base point free theorem, there exists an integer $K \in \mathbb{Z}^{+}$such that an orthonormal basis of $K\left(K_{\bar{M}}+D\right)$ gives a holomorphic map

$$
\Phi: \bar{M} \rightarrow \mathbb{C P}^{N}
$$

Date: June 22, 2021. 
where $N=\operatorname{dim} H^{0}\left(\bar{M}, K\left(K_{\bar{M}}+D\right)\right)-1$. The mainly result in this article is that the completion of $(U, \omega)$ in the sense of Theorem 1 homeomorphic to $\Phi(\bar{M} \backslash D)$. If the divisor $D$ is simple normal crossing with coefficients 1 , then there is a similar result. To dealing with this problem, the continuity method is taken proposed by G. La Nave and G. Tian in [18.

To begin with, let $\bar{M}$ be a projective manifold with a Kähler metric $\omega_{0}$ and $D$ be a smooth hypersurface in $\bar{M}$ such that $K_{\bar{M}}+D$ is big and semi-ample. $h_{D}$ is denoted by the hermitian metric on $L_{D}$, the associated line bundle of $D$ such that $\omega_{0}-\sqrt{-1} \partial \bar{\partial} \log \log ^{2}\left|s_{D}\right|_{h_{D}}^{2}>0$, where $s_{D}$ is the defining section of $D$. The following 1-parameter family equations are considered:

$$
(1+t) \omega=\omega_{0}-\sqrt{-1} \partial \bar{\partial} \log \log ^{2}\left|s_{D}\right|_{h_{D}}^{2}-t(\operatorname{Ric}(\omega)-[D]),
$$

where $[D]$ is the current of integratioin along $D$.

Recall that $\omega$ is sald to have cusp singularities along $D$ if, whenever $D$ is locally given by $\left(z_{1}=0\right)$, $\omega$ is quasi-isometric to the cusp metric

$$
\omega_{\text {cusp }}=\frac{\sqrt{-1} d z_{1} \wedge d \overline{z_{1}}}{\left|z_{1}\right|^{2} \log ^{2}\left|z_{1}\right|^{2}}+\sum_{k=2}^{n} d z_{k} \wedge d \overline{z_{k}} .
$$

Since $\omega_{0}-\sqrt{-1} \partial \bar{\partial} \log \log ^{2}\left|s_{D}\right|_{h_{D}}^{2}$ is a Kähler metric on $\bar{M} \backslash D$ with cusp singularities, the equation (1.2) essentially state the variation of cusp Kähler metric along $t$. Therefore, the equation (1.2) is called the cusp continuity equation.

Theorem 1.3. The cusp continuity equation (1.2) is solvable for all $t \in[0,+\infty)$.

$\omega_{t}$ is denoted by the solution of (1.2), then we have the following convergence result.

Theorem 1.4. $\omega_{t}$ converge to a unique weakly Kähler metric $\omega_{1}$ such that $\omega_{1}$ is smooth on $\bar{M} \backslash(D \cup \mathcal{S})$ and satisfies

where

$$
\operatorname{Ric}\left(\omega_{1}\right)=-\omega_{1} \text {, on } \bar{M} \backslash\left(\mathcal{S}_{\bar{M}} \cup D\right),
$$

$$
\mathcal{S}_{\bar{M}}=\bigcap\left\{E \mid E \text { is an effective disivior such that } K_{\bar{M}}+D-\rho E>0 \text { for some } \rho>0\right\} .
$$

If $G$ is a big divisor, we denoted $B_{+}(G)$ by the intersection of all effective $\mathbb{Q}$-divisors $E$ such that $G-E$ is ample. Then $\mathcal{S}_{\bar{M}}$ appeared in Theorem 1.4 is $B_{+}\left(K_{\bar{M}}+D\right)$. Observing that $\Phi:(\bar{M}, D) \rightarrow$ $(\Phi(\bar{M}), \Phi(D))$ can be viewed as a resolution of $(\Phi(\bar{M}), \Phi(D))$ and $K_{\bar{M}}+D=\Phi^{*}\left(K_{\Phi(\bar{M})}+\Phi(D)\right)$. According to Theorem 0-3-12 [15], $K_{\Phi(\bar{M})}+\Phi(D)$ is ample. Let $\Phi(\bar{M})_{\text {reg }}$ be the regular part of $\Phi(\bar{M})$ and $\Phi(\bar{M})_{\text {sing }}$ be the singular part of $\Phi(\bar{M})$. The following Proposition illustrate the connection between $B_{+}\left(K_{\bar{M}}+D\right)$ and $\Phi(\bar{M})_{\text {sing }}$, due to Proposition 2.3 [3].

Proposition 1.5. Let $\pi: X \rightarrow Y$ be a birational morphism between normal projective varieties. For any big $\mathbb{R}$-divisor $G$ on $Y$ and any effective $\pi$-exceptional divisor $\mathbb{R}$-divisor $F$ on $X$, then we have

$$
B_{+}\left(\pi^{*} G+F\right)=\pi^{-1}\left(B_{+}(G)\right) \cup \operatorname{Exc}(\pi),
$$

where $\operatorname{Exc}(\pi) \subset X$ is the set of points $x \in X$ such that $\pi$ is not biregular.

From Theorem 1.4 and Proposition [1.5, the following Corollary is derived immediately.

Corollary 1.6.

$$
\mathcal{S}_{\bar{M}}=B_{+}\left(K_{\bar{M}}+D\right)=\Phi^{-1}\left(B_{+}\left(K_{\Phi(\bar{M})}+\Phi(D)\right)\right) \cup \operatorname{Exc}(\Phi)=\Phi^{-1}\left(\Phi(\bar{M})_{\text {sing }}\right)
$$


and

$$
\bar{M} \backslash\left(\mathcal{S}_{\bar{M}} \cup D\right)=\bar{M}_{\text {reg }} \backslash D,
$$

where $\bar{M}_{\text {reg }}$ represents $\Phi^{-1}\left(\Phi(\bar{M})_{\text {reg }}\right)$. Furthermore the metric $\omega_{1}$ is smooth on $\bar{M}_{\text {reg }} \backslash D$.

Remark 1.7. If the codimension of $\Phi(D)$ is not 1 , then $D$ is an exceptional divisor of the resolution $\Phi$. Thus, $\bar{M} \backslash\left(\mathcal{S}_{\bar{M}} \cup D\right)=\bar{M}_{\text {reg }}$.

$M$ is denoted by $\bar{M} \backslash D$. The next result states that the limit space $\left(M, \omega_{t}\right)$ converge to in the Gromov-Hausdorff topology has more regular properties, such as metric structure, algebraic structure.

Theorem 1.8. The following results are hold.

(1) $\left(M, \omega_{t}, x\right)$ converges in the Gromov-Hausdorff topology to a length space $\left(M_{1}, d_{1}, x_{1}\right)$ which is the metric completion $\left(\bar{M}_{r e g} \backslash D, \omega_{1}\right)$.

(2) $M_{1}=\mathcal{R} \cup \mathcal{S}$ and $\mathcal{R}=\bar{M}_{\text {reg }} \backslash D$, where $\mathcal{R}$ is the regular part and $\mathcal{S}$ is the singular part.

(3) $\mathcal{R}$ is geodesically convex and $\mathcal{S}$ is closed set which has codimension $\geq 2$.

(4) $M_{1}$ homeomorphic to a normal quasi-subvariety $\Phi(\bar{M} \backslash D)$.

Acknowledgement:The author would like to thank Professor Gang Tian for his constant help, support and encouragement.

\section{Preliminaries}

In this section, we list some fundamental definitions and results which will be used in the later.

Definition 2.1. Let $V$ be an open set in $\mathbb{C}^{n}$. A holomorphic map from $V$ into a complex manifold $M$ of complex dimension $n$ is called a quasi-coordinate map if it is of maximal rank everywhere in $V$. This open set $V$ is called a local quasi-coordinate of $M$.

Definition 2.2. Let $M$ be a complete Kähler manifold and $\omega$ is the Kähler form. $(M, \omega)$ is called bounded geometry if there is a quasi-coordinates $\Gamma=\left\{\left(V ; v^{1}, \cdots, v^{n}\right)\right\}$ which satisfies the following three conditions:

(1) $M$ is covered by the image of $\left(V ; v^{1}, \cdots, v^{n}\right)$.

(2) The complement of some open neighborhood of $D$ is covered by a finite of $\left(V ; v^{1}, \cdots, v^{n}\right)$ which are local coordinates in the usual sense.

(3) There exist positive constants $C$ and $A_{k}(k=0,1,2, \cdots)$ independent of $\Gamma$ such that at each $\left(V ; v^{1}, \cdots, v^{n}\right)$, the inequalities

$$
\begin{gathered}
\frac{1}{C} \delta_{i j}<\left(g_{i \bar{j}}\right)<C \delta_{i j}, \\
\left|\frac{\partial^{|p|+|q|}}{\partial v^{|p|} \partial \bar{v}|q|} g_{i \bar{j} \mid}\right|<A_{|p|+|q|}, \text { for any multiindices } p \text { and } q
\end{gathered}
$$

hold, where $g_{i \bar{j}}$ denote the component of $\omega$ with respect to $V$.

Now we define the Hölder space of $C^{k, \lambda}$-functions on a complete Kähler manifold $(M, \omega)$ which cover by the image of quasi-coordinates. For a nonnegative integer $k, \lambda \in(0,1)$ and $u \in C^{k}(M)$, we 
define

$$
\begin{aligned}
& \|u\|_{k, \lambda}=\sup _{V \in \Gamma}\left\{\sup _{z \in V}\left(\sum_{|p|+|q| \leq k}\left|\frac{\partial^{|p|+|q|}}{\partial v^{|p|} \partial \bar{v}^{|q|}} u(z)\right|\right)\right. \\
& \left.+\sup _{z, z^{\prime} \in V}\left(\sum_{|p|+|q|=k}\left|z-z^{\prime}\right|^{-\lambda}\left|\frac{\partial^{|p|+|q|}}{\partial v^{|p|} \partial \bar{v}^{|q|}} u(z)-\frac{\partial^{|p|+|q|}}{\partial v^{|p|} \partial \bar{v}^{|q|}} u\left(z^{\prime}\right)\right|\right)\right\} .
\end{aligned}
$$

The function space $C^{k, \lambda}(M)$ is, by definition,

$$
C^{k, \lambda}(M)=\left\{u \in C^{k}(M) ;\|u\|_{k, \lambda}<\infty\right\},
$$

which is a Banach space with respect to the norm $\|\cdot\|_{k, \lambda}$.

Next we state the generalized maximum principle, due to Yau (Proposition 1.6 [9]).

Theorem 2.3. Suppose $(M, \omega)$ is a complete Kähler manifold with bounded geometry and $f$ is a function on $M$ which is bounded from above. Then there exists a sequence $x_{i}$ in $M$ such that $\lim _{i \rightarrow \infty} f\left(x_{i}\right)=\sup f, \lim _{i \rightarrow \infty}\left|\nabla f\left(x_{i}\right)\right|=0$ and $\varlimsup_{i \rightarrow \infty} \operatorname{Hess}(f)\left(x_{i}\right) \leq 0$, where the Hessian is taken with respect to $\omega$.

Now we introduce the Bochner formula on a general line bundle. Let $(M, \omega)$ be a Kähler manifold of dimension $n$ and $(L, h)$ be a Hermitian Line bundle over $M$. Let $\Theta_{h}$ be the Chern curvature form of $h$. Let $\nabla$ and $\bar{\nabla}$ denote the $(1,0)$ and $(0,1)$ part of a connection respectively. The connection appeared in this paper is usually known as the Chern connection or Levi-Civita connection.

For a holomorphic section $\tau \in H^{0}(M, L)$ we write for simplicity

$$
|\tau|=|\tau|_{h},|\nabla \tau|_{h \otimes \omega}=|\nabla \tau|
$$

and

$$
|\nabla \nabla \tau|^{2}=\sum_{i, j}\left|\nabla_{i} \nabla_{j} \tau\right|^{2},|\bar{\nabla} \nabla \tau|^{2}=\sum_{i, j}\left|\nabla_{\bar{i}} \nabla_{j} \tau\right|^{2} .
$$

By direct computation we have

Lemma 2.4. (Bochner formulas). For any $\tau \in H^{0}(M, L)$ one has

$$
\triangle_{\omega}|\tau|^{2}=|\nabla \tau|^{2}-|\tau|^{2} \cdot \operatorname{tr}_{\omega} \Theta_{h}
$$

and

$$
\begin{aligned}
\triangle_{\omega}|\nabla \tau|^{2}=|\nabla \nabla \tau|^{2}+|\bar{\nabla} \nabla \tau|^{2}-\nabla_{j}\left(\Theta_{h}\right)_{i \bar{j}}\left\langle\tau, \nabla_{\bar{i}} \bar{\tau}\right\rangle-\nabla_{\bar{j}}\left(\operatorname{tr}_{\omega} \Theta_{h}\right)\left\langle\nabla_{j} \tau, \bar{\tau}\right\rangle \\
\quad+R_{i \bar{j}}\left\langle\nabla_{j} \tau, \nabla_{\bar{i}} \bar{\tau}\right\rangle-2\left(\Theta_{h}\right)_{i \bar{j}}\left\langle\nabla_{j} \tau, \nabla_{\bar{i}} \bar{\tau}\right\rangle-|\nabla \tau|^{2} \cdot \operatorname{tr}_{\omega} \Theta_{h}
\end{aligned}
$$

where $R_{i \bar{j}}$ is the Ricci curvature of $\omega,\langle$,$\rangle is the inner product defined by h$.

\section{EXISTENCE AND UNIQUENESS OF CUSP CONTINUITY EQUATION}

This section is devoted to prove the Theorem 1.3. When $t=0$ the equation (1.2) has a solution $\omega(0)=\omega_{0}-\sqrt{-1} \partial \bar{\partial} \log \log ^{2}\left|s_{D}\right|_{h_{D}}^{2}$. For a fixed $t \neq 0$, we reduce (1.2) to a scalar equation. First, the background metric will be constructed. Since $K_{\bar{M}}+D$ is semi-ample, the Kawamata base point free Theorem claims that there exists an integer $K_{0}$ such that $K_{0}\left(K_{\bar{M}}+D\right)$ has no base point. Then a basis of $H^{0}\left(\bar{M}, K_{0}\left(K_{\bar{M}}+D\right)\right)$ gives a holomorphic map

$$
\Phi: \bar{M} \rightarrow \mathbb{C P}^{N}
$$


where $N=\operatorname{dim} H^{0}\left(\bar{M}, K_{0}\left(K_{\bar{M}}+D\right)\right)-1$. $\omega_{F S}$ is denoted by the Fubini-Study metric on $\mathbb{C P}^{N}$. Set $\eta_{1}=\frac{1}{K_{0}} \Phi^{*}\left(\omega_{F S}\right)$. Since $\eta_{1} \in c_{1}\left(K_{\bar{M}}+D\right)$, there exist a smooth volume form $\Omega$ on $\bar{M}$ and hermitian metric $h_{D}^{t}$ on $L_{D}$ such that $\eta_{1}=-\operatorname{Ric}(\Omega)+\Theta_{h_{D}^{t}}$ and $\frac{1}{1+t^{\prime}} \omega_{0}+\frac{t^{\prime}}{1+t^{\prime}} \eta_{1}-\sqrt{-1} \partial \bar{\partial} \log \log ^{2}\left|s_{D}\right|_{h_{D}^{t}}>0$ for $t^{\prime} \in[0, t]$, where $\Theta_{h_{D}^{t}}$ is the curvature form of $L_{D}$ with the metric $h_{D}^{t}$. Set $l=\frac{t}{1+t}$, then $\widetilde{\omega}_{l}:=$ $(1-l) \omega_{0}+l \eta_{1}-\sqrt{-1} \partial \bar{\partial} \log \log ^{2}\left|s_{D}\right|_{h_{D}^{t}}$ is chosen as the background meric. Therefore the equation (1.2) is reduced to the following scalar equation

$$
\left(\widetilde{\omega}_{l}+\sqrt{-1} \partial \bar{\partial} u_{l}\right)^{n}=e^{\frac{u_{l}}{l}} \frac{\Omega_{l}}{\left|s_{D}\right|_{h_{D}^{l}}^{2} \log ^{2}\left|s_{D}\right|_{h_{D}^{l}}^{2}},
$$

where $\left|s_{D}\right|_{h_{D}^{l}}^{2}$ is denoted by $\left|s_{D}\right|_{h_{D}^{t}}^{2}$ and $\Omega_{l}=\Omega\left(\frac{\log ^{2}\left|s_{D}\right|_{h_{D}}^{2}}{\log ^{2}\left|s_{D}\right|_{h_{D}^{l}}^{2}}\right)^{\frac{1-l}{l}}$ is a smooth volume form on $\bar{M}$. For the convenience, we simplified the notation of the above equation as following

$$
\left(\widetilde{\omega}_{l}+\sqrt{-1} \partial \bar{\partial} u_{l}\right)^{n}=e^{\frac{u_{l}}{l}} \frac{\Omega_{l}}{\left|s_{D}\right|^{2} \log ^{2}\left|s_{D}\right|^{2}} .
$$

To get a complete metric, we define an open subset $U$ in $C^{k, \lambda}(M)$ by

$$
U=\left\{v \in C^{k, \lambda}(M) \mid \frac{1}{C} \widetilde{\omega}_{l}<\widetilde{\omega}_{l}+\sqrt{-1} \partial \bar{\partial} v<C \widetilde{\omega_{l}}, \text { for some positive constant } C\right\},
$$

where $M=\bar{M} \backslash D$. If $u_{l}$ belongs to $U$ and satisfies (3.1), then $\widetilde{\omega}_{l}+\sqrt{-1} \partial \bar{\partial} u_{l}$ is a complete Kähler metric.

Now we take the continuity method to solve the equation (3.1). Consider the following equations

$$
\left(\widetilde{\omega}_{l}+\sqrt{-1} \partial \bar{\partial} u_{l, s}\right)^{n}=e^{\frac{u_{l, s}}{l}} \cdot e^{s F} \cdot \widetilde{\omega}_{l}^{n},
$$

where $F=\frac{\Omega_{l}}{\widetilde{\omega_{l}{ }^{n}\left|s_{D}\right|^{2} \log ^{2}\left|s_{D}\right|^{2}}}$. We consider

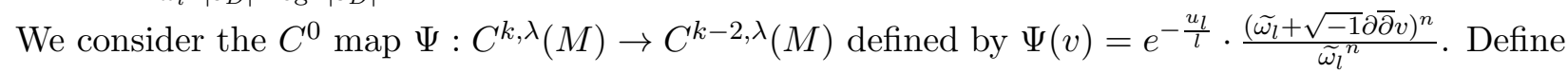

$$
S=\left\{s \in[0,1] \mid \text { there is a solution } u_{l, s} \text { satisfies } \Psi\left(u_{l, s}\right)=e^{s F}\right\} .
$$

Obviously, $0 \in S$. To prove $1 \in S$, it is sufficient to show that $S$ is open and closed. The inverse mapping theorem implies the openness. The Fréchet derivative $\Psi^{\prime}\left(u_{l, s}\right): C^{k, \lambda}(M) \rightarrow C^{k-2, \lambda}(M)$ at $u_{l, s} \in U$ is given by

$$
h \rightarrow e^{s F}\left(\triangle_{\omega_{l, s}} h-\frac{h}{l}\right),
$$

where $\omega_{l, s}=\widetilde{\omega}_{l}+\sqrt{-1} \partial \bar{\partial} u_{l, s}$. Due to Kobayashi [16], $F \in C^{k-2, \lambda}(M)$. Therefore, we have to show that, for any $w \in C^{k-2, \lambda}(M)$,

$$
\triangle_{\omega_{l, s}} h-\frac{h}{l}=w
$$

can be solved for $h \in C^{k, \lambda}(M)$ and that $|h|_{C^{k, \lambda}} \leq C|w|_{C^{k-2, \lambda}}$ for some constant $C$ independent of $w$.

We first to show that there is at most one function $h$ in $C^{k, \lambda}(M)$ solving the equation (3.3). It suffices to verify that $\triangle_{\omega_{l, s}} h-\frac{h}{l}=0$ and $h \in C^{k, \lambda}(M)$ imply $h \equiv 0$. Note that $\omega_{l, s}$ is complete Kähler metric with bounded geometry due to Lemma 2 [16] and Proposition 1.4 [9]. For such a metric we can use the generalized maximum principle. Suppose $h \in C^{k, \lambda}(M), h$ is in particular bounded. The generalized maximum principle implies that there exists a sequence of points $\left\{x_{i}\right\}$ in $M$ such that 
$\lim _{i \rightarrow \infty} h\left(x_{i}\right)=\sup h$ and $\varlimsup_{i \rightarrow \infty} \triangle_{\omega_{l, s}} h\left(x_{i}\right) \leq 0$. We immediately see that sup $h \leq 0$ according to the equation $\triangle_{\omega_{l, s}} h-\frac{h}{l}=0$. Similarly, inf $h \geq 0$ and $h \equiv 0$.

Now we prove the existence of $h$. Let $\left\{\Omega_{i}\right\}$ be an exhaustion of $M$ by compact subdomains. Suppose $w \in C^{k-2, \lambda}(M)$ and let $h_{i}$ be the unique solution to

$$
\begin{gathered}
\triangle_{\omega_{l, s}} h_{i}-\frac{h_{i}}{l}=w \text { in } \Omega_{i}, \\
h_{i}=0 \text { on } \partial \Omega_{i} .
\end{gathered}
$$

The maximum principle applied to $\Omega_{i}$ shows that

$$
\sup _{\Omega_{i}}\left|h_{i}\right| \leq l \cdot \sup _{\Omega_{i}}|w|
$$

Interior Schauder estimates shows that a sequence of $h_{i}$ converge to some $h \in C^{k, \lambda}(M)$ which solves the equation (3.3) and that the estimate $|h|_{C^{k, \lambda}} \leq C|w|_{C^{k-2, \lambda}}$.

Next, it remains to show that $S$ is closed. Assume that $\left\{s_{i}\right\} \subset E$ is a sequence with $\lim _{i \rightarrow \infty} s_{i}=\bar{s}$ and $u_{l, s_{i}}$ is the solution of (3.2) with $s=s_{i}$. We want to prove $\bar{s} \in E$. It amounts to getting a prior $C^{k, \lambda}(M)$-estimate for each $u_{l, s_{i}}$. By applying the generalized maximum principle to (3.2), we have

$$
\sup _{M}\left|u_{l, s_{i}}\right| \leq l \cdot s_{i} \sup _{M}|F| \leq C \sup _{M}|F| .
$$

So we have the $C^{0}$-estimate due to Lemma 1 [16]. For the $C^{2}$-estimate, since $\left(M, \widetilde{\omega_{l}}\right)$ is a bounded geometry, by the standard calculation we have

$$
\operatorname{Ric}\left(\omega_{l, s_{i}}\right)=-\frac{1}{l} \omega_{l, s_{i}}+\frac{1}{l} \widetilde{\omega}_{l}-s_{i} \sqrt{-1} \partial \bar{\partial} F+\operatorname{Ric}\left(\widetilde{\omega}_{l}\right) \leq-\frac{1}{l} \omega_{l, s_{i}}+C \widetilde{\omega}_{l}
$$

and

Then

$$
\triangle_{\omega_{l, s_{i}}} \log \operatorname{tr}_{\widetilde{\omega}_{l}} \omega_{l, s_{i}} \geq \frac{1}{\operatorname{tr}_{\widetilde{\omega_{l}}} \omega_{l, s_{i}}}\left(-g^{i \bar{j}}\left(\widetilde{\omega}_{l}\right) R_{i \bar{j}}\left(\omega_{l, s_{i}}\right)+g^{i \bar{j}}\left(\omega_{l, s_{i}}\right) g_{k \bar{l}}\left(\omega_{l, s_{i}}\right) R_{i \bar{j}} k \bar{l}\left(\widetilde{\omega}_{l}\right)\right) .
$$

$$
\triangle_{\omega_{l, s_{i}}} \log \operatorname{tr}_{\widetilde{\omega}_{l}} \omega_{l, s_{i}} \geq-\operatorname{atr}_{\omega_{l, s_{i}}} \widetilde{\omega}_{l}-\frac{A}{\operatorname{tr}_{\widetilde{\omega_{l}}} \omega_{l, s_{i}}}-C,
$$

where $-a$ is the lower bound of holomorphic bisectional curvature of metric $\widetilde{\omega_{l}}$. Note that

$$
\triangle_{\omega_{l, s_{i}}} u_{l, s_{i}}=n-\operatorname{tr}_{\omega_{l, s_{i}}} \widetilde{\omega}_{l} .
$$

Let $H=\log \operatorname{tr}_{\widetilde{\omega}_{l}} \omega_{l, s_{i}}-(a+1) u_{l, s_{i}}$, then

$$
\triangle_{\omega_{l, s_{i}}} H \geq \operatorname{tr}_{\omega_{l, s_{i}}} \widetilde{\omega}_{l}-\frac{A}{\operatorname{tr}_{\widetilde{\omega_{l}}} \omega_{l, s_{i}}}-C .
$$

By the generalized maximum principle, there exists a sequence $\left\{x_{i}\right\}$ such that $\lim _{i \rightarrow \infty} H\left(x_{i}\right)=\sup H$ and $\overline{\lim }_{i \rightarrow \infty} \sqrt{-1} \partial \bar{\partial} H\left(x_{i}\right) \leq 0$. So we have a subsequence also denoted by $\left\{x_{i}\right\}$ such that

$$
\lim _{i \rightarrow \infty} \operatorname{tr}_{\widetilde{\omega}_{l}} \omega_{l, s_{i}}\left(\operatorname{tr}_{\omega_{l, s_{i}}} \widetilde{\omega}_{l}-C\right)\left(x_{i}\right) \leq A .
$$

Nota that $\left(\operatorname{tr}_{\widetilde{\omega_{l}}} \omega_{l, s_{i}}\right)^{\frac{1}{n-1}} \leq C^{\prime} \operatorname{tr}_{\omega_{l, s_{i}}} \widetilde{\omega}_{l}$. Then we get

If

$$
\lim _{i \rightarrow \infty} \operatorname{tr}_{\widetilde{\widetilde{\omega}_{l}}} \omega_{l, s_{i}}\left(\frac{1}{C^{\prime}}\left(\operatorname{tr}_{\widetilde{\omega_{l}}} \omega_{l, s_{i}}\right)^{\frac{1}{n-1}}-C\right)\left(x_{i}\right) \leq A .
$$

$$
\lim _{i \rightarrow \infty}\left(\operatorname{tr}_{\widetilde{\omega}_{l}} \omega_{l, s_{i}}\right)^{\frac{1}{n-1}}\left(x_{i}\right) \leq 2 C^{\prime} C,
$$


then we see

$$
\lim _{i \rightarrow \infty} \operatorname{tr}_{\widetilde{\omega_{l}}} \omega_{l, s_{i}}\left(x_{i}\right) \leq C
$$

Otherwise

$$
\lim _{i \rightarrow \infty}\left(\operatorname{tr}_{\widetilde{\omega_{l}}} \omega_{l, s_{i}}\right)^{\frac{1}{n-1}}\left(x_{i}\right) \geq 2 C^{\prime} C
$$

then by (3.4) we have

$$
\lim _{i \rightarrow \infty} \operatorname{tr}_{\widetilde{\omega_{l}}} \omega_{l, s_{i}}\left(x_{i}\right) \leq C .
$$

Therefore, $H \leq C$. This implies $\operatorname{tr}_{\widetilde{\omega_{l}}} \omega_{l, s_{i}} \leq C$. Furthermore by a standard inequality, we get $C^{-1} \widetilde{\omega}_{l} \leq$ $\omega_{l, s_{i}} \leq C \widetilde{\omega}_{l}$.

For the 3-order estimate, let $T=\left|\nabla_{\widetilde{g}_{l}} \partial \bar{\partial} u_{l, s_{i}}\right|_{g_{l, s_{i}}}^{2}$, where $\widetilde{g}_{l}$ and $g_{l, s_{i}}$ represent Riemannian metrics associated with Kähler forms $\widetilde{\omega}_{l}$ and $\omega_{l, s_{i}}$. By a standard computations (c.f. Proposition 4.3 [9]), we have

$$
\triangle_{\omega_{l, s_{i}}}\left(T+C \triangle_{\widetilde{\omega_{l}}} u_{l, s_{i}}\right) \geq C_{1} T-C_{2} .
$$

By the Laplace estimate of $u_{l, s_{i}}$ and generalized maximum principle, we get $T \leq C$. Thus, by taking a subsequence if necessary, $u_{l, s_{i}} C^{2, \lambda}$-converge to a solution with $s=\bar{s}$. This implies $S$ is closed.

Next we prove the uniqueness of equation (3.1). Suppose that $u_{l, 1}$ and $u_{l, 2}$ are solutions to (3.1). Set $\omega_{2}=\widetilde{\omega}_{l}+\sqrt{-1} \partial \bar{\partial} u_{l, 2}$, then we have

$$
\frac{\left(\omega_{2}+\sqrt{-1} \partial \bar{\partial}\left(u_{l, 1}-u_{l, 2}\right)\right)^{n}}{\omega_{2}^{n}}=e^{\frac{u_{l, 1}-u_{l, 2}}{l}} .
$$

Since $\left(M, \omega_{2}\right)$ is a complete Kähler manifold with bounded geometry (c.f. Proposition 1.4 [9]), applying the generalized maximum principle, there exists a sequence $\left\{x_{i}\right\}$ such that $\lim _{i \rightarrow \infty}\left(u_{l, 1}-u_{l, 2}\right)\left(x_{i}\right)=$ $\sup _{M}\left(u_{l, 1}-u_{l, 2}\right)$ and $\varlimsup_{i \rightarrow \infty} \operatorname{Hess}\left(u_{l, 1}-u_{l, 2}\right)\left(x_{i}\right) \leq 0$. Furthermore, we obtain $u_{l, 1} \leq u_{l, 2}$. By the same argument, we have $u_{l, 1} \geq u_{l, 2}$. Therefore, the equation (3.1) has only one solution. Finally, the cusp continuity equation is solvable for all $t \in[0, \infty)$ i.e., $l \in[0,1)$.

\section{Convergence of CUSP COntinuity EQuation}

In this section we investigate the regular properties of limit metric.

Lemma 4.1. Let $F$ be a divisor on $\bar{M}$. If $F$ is nef and big, then there is an effective divisor $E=$ $\sum_{i} a_{i} E_{i}$ such that $F-\epsilon E>0$ for all sufficiently small $\epsilon>0$.

By the assumption that $K_{\bar{M}}+D$ is big and semi-ample, there exists an effective divisor $E=\sum_{i} a_{i} E_{i}$ such that $K_{\bar{M}}+D-\epsilon E>0$ for all sufficiently small $\epsilon>0$ according to Lemma 4.1. Thus we choose a volume form $\Omega$, a hermitian metric $h_{D}^{\prime}$ on $L_{D}$ and hermitian metrics $h_{E_{i}}$ such that

$$
-\operatorname{Ric}(\Omega)+\Theta_{D}^{\prime}-\sum_{i} \epsilon a_{i} \Theta_{E_{i}}>0
$$

where $\Theta_{D}^{\prime}$ and $\Theta_{E_{i}}$ represent curvature forms of line bundles $L_{D}$ and $L_{E_{i}}$ associated with metrics $h_{D}^{\prime}$ and $h_{E_{i}}$ respectively. $s_{D}$ and $s_{E_{i}}$ are denoted by the defining sections of $L_{D}$ and $L_{E_{i}}$. For simplicity, we write $\log \left|s_{E}\right|^{2}=\sum_{i} a_{i} \log \left|s_{E_{i}}\right|^{2}$. By taking appropriate $\Omega, h_{D}^{\prime}$ and $h_{E_{i}}$, we can assume that

$$
-\operatorname{Ric}(\Omega)+\Theta_{D}^{\prime}-\sum_{i} \epsilon a_{i} \Theta_{E_{i}}>0
$$

and

$\widetilde{\omega_{l, E}}:=(1-l) \omega_{0}+l\left(-\operatorname{Ric}(\Omega)-\sqrt{-1} \partial \bar{\partial} \log \left|s_{D}\right|_{h_{D}^{\prime}}^{2}+\epsilon \sqrt{-1} \partial \bar{\partial} \log \left|s_{E}\right|^{2}\right)-\sqrt{-1} \partial \bar{\partial} \log \log ^{2}\left|s_{D}\right|_{h_{D}^{\prime}}^{2}>0$ 
for $l \in\left[\frac{1}{2}, 1\right]$. Let $\widetilde{\omega}_{l}:=(1-l) \omega_{0}+l\left(-\operatorname{Ric}(\Omega)+\Theta_{D}\right)-\sqrt{-1} \partial \bar{\partial} \log \log ^{2}\left|s_{D}\right|_{h_{D}}^{2}$ (may not be a metric), where the hermitian metric $h_{D}$ is defined as $\omega(0)=\omega_{0}-\sqrt{-1} \partial \bar{\partial} \log \log ^{2}\left|s_{D}\right|_{h_{D}}^{2}>0$ and $\Theta_{D}$ is the curvature form of $h_{D}$. Then the equation (1.2) is written as

$$
\left(\widetilde{\omega_{l}}+\sqrt{-1} \partial \bar{\partial} u_{l}\right)^{n}=e^{\frac{u_{l}}{l}} \cdot \frac{\Omega}{\left|s_{D}\right|_{h_{D}}^{2} \log ^{2}\left|s_{D}\right|_{h_{D}}^{2}} .
$$

This equation is also equivalent to

$$
\left(\widetilde{\omega_{l, E}}+\sqrt{-1} \partial \bar{\partial} w_{l}\right)^{n}=e^{\frac{1}{l} w_{l}+\epsilon \log \left|s_{E}\right|^{2}} \cdot \frac{\Omega_{l}}{\left|s_{D}\right|_{h_{D}^{\prime}}^{2} \log ^{2}\left|s_{D}\right|_{h_{D}^{\prime}}^{2}},
$$

where $w_{l}=u_{l}-l \epsilon \log \left|s_{E}\right|^{2}+l \log \frac{\left|s_{D}\right|_{h_{D}^{\prime}}^{2}}{\left|s_{D}\right|_{h_{D}}^{2}}+\log \frac{\log ^{2}\left|s_{D}\right|_{h_{D}^{\prime}}^{2}}{\log ^{2}\left|s_{D}\right|_{h_{D}}^{2}}$ and $\Omega_{l}=\Omega\left(\frac{\log ^{2}\left|s_{D}\right|_{h_{D}}^{2}}{\log ^{2}\left|s_{D}\right|_{h_{D}^{\prime}}^{2}}\right)^{\frac{1-l}{l}}$.

Lemma 4.4. There exists a constant $C$ independent of $l$ such that $-C \leq w_{l} \leq C-l \epsilon \log \left|s_{E}\right|^{2}$.

Proof. For the lower bound, we note that $\widetilde{\omega_{l, E}}=(1-l) \omega_{0}+l\left(-\operatorname{Ric}(\Omega)+\Theta_{D}^{\prime}-\sum_{i} \epsilon a_{i} \Theta_{E_{i}}\right)-$ $\sqrt{-1} \partial \bar{\partial} \log \log ^{2}\left|s_{D}\right|_{h_{D}^{\prime}}^{2}$ is a complete Kähler metric with bounded geometry on $M$. Applying the generalized maximum principle to (4.3), we get $w_{l} \geq-C-l \epsilon \log \left|s_{E}\right|^{2} \geq-C$.

For the upper bound, we differentiate $l$ at both side of equation (4.2), then

$$
\triangle_{\omega_{l}} \dot{u}_{l}+\frac{n}{l}-\frac{1}{l} \triangle_{\omega_{l}} u_{l} \geq \frac{1}{l}\left(\dot{u}_{l}-\frac{u_{l}}{l}\right) .
$$

where $\omega_{l}=\widetilde{\omega}_{l}+\sqrt{-1} \partial \bar{\partial} u_{l}$.

By the simple calculation, we get

$$
\triangle_{\omega_{l}}\left(\frac{u_{l}}{l}-n \log l\right)^{\prime} \geq \frac{1}{l}\left(\frac{u_{l}}{l}-n \log l\right)^{\prime} .
$$

According to the generalized maximum principle, $\left(\frac{u_{l}}{l}-n \log l\right)$ decrease when $l$ tends to 1 . Therefore, there exists a constant $C$ such that $u_{l} \leq C$. By the definition of $w_{l}$, we see $w_{l} \leq C-l \epsilon \log \left|s_{E}\right|^{2}$.

Lemma 4.5. There exist two constants $C$ and a independent of $l$ such that $C^{-1}\left|s_{E}\right|^{2 l \epsilon(a+1)} \widetilde{\omega_{l, E}} \leq$ $\omega_{l}:=\widetilde{\omega_{l}}+\sqrt{-1} \partial \bar{\partial} u_{l} \leq C\left|s_{E}\right|^{-2 l \epsilon(a+1)(n-1)} \widetilde{\omega_{l, E}}$.

Proof. Since Ric $\left(\omega_{l}\right) \geq-\frac{1}{l} \omega_{l}$, by Yau's Schwarz Lemma [25], we have

$$
\triangle_{\omega_{l}} \log \operatorname{tr}_{\omega_{l}} \widetilde{\omega_{l, E}} \geq-a \cdot \operatorname{tr}_{\omega_{l}} \widetilde{\omega_{l, E}}-\frac{1}{l}
$$

where $a$ is a positive upper bound of the holomorphic bisectional curvature of $\widetilde{\omega_{l, E}}$ for $l \in[0,1]$. Put $H=\log \operatorname{tr}_{\omega_{l}} \widetilde{\omega_{l, E}}-(a+1) w_{l}$, then we get

$$
\triangle_{\omega_{l}} H \geq \operatorname{tr}_{\omega_{l}} \widetilde{\omega_{l, E}}-C .
$$

By the generalized maximum principle, there exists a sequence $\left\{x_{i}\right\}$ such that $\lim _{i \rightarrow \infty} H\left(x_{i}\right)=\sup _{M} H$ and $\varlimsup_{i \rightarrow \infty} \triangle_{\omega_{l}} H\left(x_{i}\right) \leq 0$. Thus by the Lemma 4.4 we have $H \leq C$. This implies

Note that

$$
\operatorname{tr}_{\omega_{l}} \widetilde{\omega_{l, E}} \leq \frac{C}{\left|s_{E}\right|^{2 l \epsilon(a+1)}} .
$$

Hence this Lemma is proved.

$$
\operatorname{tr}_{\widetilde{\omega_{l, E}}} \omega_{l} \leq \frac{1}{(n-1) !}\left(\operatorname{tr}_{\omega_{l}} \widetilde{\omega_{l, E}}\right)^{n-1} \cdot \frac{\omega_{l}^{n}}{\widetilde{\omega_{l, E}} n} \leq \frac{C}{\left|s_{E}\right|^{2 l \epsilon(a+1)(n-1)}} .
$$


According to Lemma 4.5, we know that for any compact subset $K \subset \bar{M} \backslash(D \cup \operatorname{Supp} E)$, there exists a constant $C_{K}>0$ independent of $l$ such that $C_{K}^{-1} \omega_{0} \leq \omega_{l} \leq C_{K} \omega_{0}$, i.e., $\left|\triangle_{\omega_{0}} u_{l}\right| \leq C_{K}$. By Theorem 17.14 in [14, we have $\left|u_{l}\right|_{C^{2, \lambda}} \leq C_{K}^{\prime}$ on $K \times\left[\frac{1}{2}, 1\right]$. Furthermore, by the standard bootstrapping argument, for any $m>0,\left|u_{l}\right|_{C^{m, \lambda}} \leq C_{K, m}$ on $K \times\left[\frac{1}{2}, 1\right]$. By the standard diagonal argument and passing to a subsequence $\left\{l_{i}\right\}$ such that $u_{l_{i}} C^{\infty}$-converge to a function on each compact $K$ when $l_{i}$ tends to 1 . The monotonicity of $\left(\frac{u_{l}}{l}-n \log l\right)$ implies that $u_{l} C^{\infty}$-converge to a function on each compact $K$ when $l$ tends to 1 . Therefore, the Theorem 1.4 is proved.

\section{Algebraic structure of the limit space}

5.1. Gromov-Hausdorff convergence: global convergence. In this subsection we consider a family of manifolds $\left(M, \omega_{l}\right)$ on which the lower bound of Ricci curvature can be controlled, i.e. $\operatorname{Ric}\left(\omega_{l}\right) \geq-\frac{1}{l} \omega_{l}$ for $l \in\left[\frac{1}{2}, 1\right)$. By Gromov precompactness theorem [4], passing to a subsequence $l_{i} \rightarrow 1$ and fix $x_{0} \in \bar{M}_{r e g} \backslash D$, we may assume that

$$
\left(M, \omega_{l_{i}}, x_{0}\right) \stackrel{d_{G H}}{\longrightarrow}\left(M_{1}, d_{1}, x_{1}\right) .
$$

The limit $\left(M_{1}, d_{1}\right)$ is a complete length metric space. It has a regular/singular decomposition $M_{1}=$ $\mathcal{R} \cup \mathcal{S}$, a point $x \in \mathcal{R}$ iff the tangent cone at $x$ is the Euclidean space $\mathbb{R}^{2 n}$. The following lemma is the same as Lemma 3.3 in [19].

Lemma 5.1. There is a sufficiently small constant $\delta>0$ such that for any $l \in\left[\frac{1}{2}, 1\right)$, if a metric ball $B_{\omega_{l}}(x, r)$ satisfies

$$
\operatorname{Vol}\left(B_{\omega_{l}}(x, r)\right) \geq(1-\delta) \operatorname{Vol}\left(B_{r}^{0}\right)
$$

where $\operatorname{Vol}\left(B_{r}^{0}\right)$ is the volume of a metric ball of radius $r$ in $2 n$-Euclidean space, then

$$
\operatorname{Ric}\left(\omega_{l}\right) \leq(2 n-1) r^{-2} \omega_{l} \text {, in } B_{\omega_{l}}(x, \delta r) .
$$

Lemma 5.2. The regular set $\mathcal{R}$ is open in the limit space $\left(M_{T}, d_{T}, x_{T}\right)$.

Proof. If $x \in \mathcal{R}$, then by Colding's volume convergence theorem [10], there exists $r=r(x)>0$ such that $\mathcal{H}^{2 n}\left(B_{d_{1}}(x, r)\right) \geq\left(1-\frac{\delta}{2}\right) \operatorname{Vol}\left(B_{r}^{0}\right)$, where $\mathcal{H}^{2 n}$ denotes the Hausdorff measure. Suppose $x_{i} \in M$ satisfying $x_{i} \stackrel{d_{G H}}{\longrightarrow} x$, then by the volume convergence theorem again, $\operatorname{Vol}_{\omega_{l_{i}}}\left(B_{\omega_{l_{i}}}\left(x_{i}, r\right)\right) \geq$ $(1-\delta) \operatorname{Vol}\left(B_{r}^{0}\right)$ for sufficiently large $i$. According to Lemma 5.1 and Anderson's harmonic radius estimate [1], there is a constant $\delta^{\prime}=\delta^{\prime}(\alpha)$ for any $0<\alpha<1$ such that the $C^{1, \alpha}$ harmonic radius at $x_{i}$ is bigger than $\delta^{\prime} \delta r$. Passing to the limit, it gives a harmonic coordinate on $B_{d_{1}}\left(x, \delta^{\prime} \delta r\right)$. This implies $B_{d_{1}}\left(x, \delta^{\prime} \delta r\right) \subset \mathcal{R}$. So $\mathcal{R}$ is open with a $C^{1, \alpha}$ Kähler metric $\overline{\omega_{1}}$; moreover $\omega_{l_{i}}$ converges to $\overline{\omega_{1}}$ in $C^{1, \alpha}$ topology on $\mathcal{R}$.

Since $\mathcal{R}$ is dense in $M_{1}$, so we have the following Lemma.

Lemma 5.3. $\left.\left(M_{1}, d_{1}\right)=\overline{\left(\mathcal{R}, \overline{\omega_{1}}\right.}\right)$, the metric completion of $\left(\mathcal{R}, \overline{\omega_{1}}\right)$.

Lemma 5.4. $\mathcal{R}$ is geodesically convex in $M_{1}$ in the sense that any minimal geodesic with endpoints in $\mathcal{R}$ lies in $\mathcal{R}$.

Proof. It is simply a consequence of Colding-Naber's Hölder continuity of tangent cones along a geodesic in $M_{1}$ [11]. Actually, if $x, y \in \mathcal{R}$, then for any minimal geodesic connecting $x$ and $y$, a neighborhood of endpoints lies in $\mathcal{R}$, so the geodesic will never touch the singular set. 
Let $\bar{D}$ be any divisor in $\bar{M}$ such that $D \cup \mathcal{S}_{\bar{M}} \subset \bar{D}$. Define the Gromov-Hausdorff limit of $\bar{D}$

$$
\overline{D_{1}}:=\left\{x \in M_{1} \mid \text { there exists } x_{i} \in \bar{D} \text { such that } x_{i} \stackrel{d_{G H}}{\longrightarrow} x\right\} .
$$

Proposition 5.5. $\left(M_{1}, d_{1}\right)$ is isometric to $\overline{\left(\bar{M} \backslash \bar{D}, \omega_{1}\right)}$, where $\omega_{1}$ is defined as Theorem 1.4.

Proof. First, we prove the following Claim.

Claim 5.6. $\overline{D_{1}} \backslash \mathcal{S}$ is a subvariety of dimension $(n-1)$ if it is not empty.

Proof. Let $x \in \overline{D_{1}} \backslash \mathcal{S}$ and $x_{i} \in \bar{D}$ such that $x_{i} \stackrel{d_{G H}}{\longrightarrow} x$. By the $C^{1, \alpha}$ convergence of $\omega_{l_{i}}$ around $x$, there are $C, r>0$ independent of $i$ and a sequence of harmonic coordinates in $B_{\omega_{l_{i}}}\left(x_{i}, r\right)$ such that $C^{-1} \omega_{E} \leq \omega_{l_{i}} \leq C \omega_{E}$ where $\omega_{E}$ is the Euclidean metric in the coordinates. Furthermore, according to Lemma 3.11 [24], any $x_{i} \in M$ converging to $x$ has a holomorphic coordinate $\left(z_{i}^{1}, z_{i}^{2}, \cdots, z_{i}^{n}\right)$ on $B_{\omega_{l_{i}}}\left(x_{i}, r\right)$ such that $C^{-1} \omega_{E}\left(\frac{\partial}{\partial z_{i}^{k}}, \frac{\partial}{\partial \bar{z}_{i}^{l}}\right) \leq \omega_{l_{i}}\left(\frac{\partial}{\partial z_{i}^{k}}, \frac{\partial}{\partial \bar{z}_{i}^{l}}\right) \leq C \omega_{E}\left(\frac{\partial}{\partial z_{i}^{k}}, \frac{\partial}{\partial \bar{z}_{i}^{l}}\right)$. Since the total volume of $\bar{D}$ is uniformly bounded for any $\omega_{l_{i}}$, the local analytic $\bar{D} \cap B_{\omega_{l_{i}}}\left(x_{i}, r\right)$ have a uniform bound of degree and so converge to an analytic set $\overline{D_{1}} \cap B_{d_{1}}(x, r)$.

From the above Claim we know that $\operatorname{dim}_{\mathbb{R}}\left(\overline{D_{1}}\right)=\operatorname{dim}_{\mathbb{R}}\left(\mathcal{S} \cup\left(\overline{D_{1}} \backslash \mathcal{S}\right)\right) \leq 2 n-2$. By the argument of [20], $\left(M_{1} \backslash \overline{D_{1}}, \overline{\omega_{1}}\right)$ homeomorphic and locally isometric to $\left(\bar{M} \backslash \bar{D}, \omega_{1}\right)$. Since $M_{1}$ is a length space and $\operatorname{dim}_{\mathbb{R}}\left(\overline{D_{1}}\right) \leq 2 n-2,\left(M_{1} \backslash \overline{D_{1}}, \overline{\omega_{1}}\right)$ isometric to $\left(\bar{M} \backslash \bar{D}, \omega_{1}\right)$. Furthermore, we have

$$
\left(M_{1}, d_{1}\right)=\overline{\left(M_{1} \backslash \overline{D_{1}}, \overline{\omega_{1}}\right)}=\overline{\left(\bar{M} \backslash \bar{D}, \omega_{1}\right)} .
$$

A direct corollary is

Corollary 5.7. $\left(M, \omega_{l}, x_{0}\right)$ converges globally to $\left(M_{1}, d_{1}, x_{1}\right)$ in the Gromov-Hausdorff topology as $l \rightarrow l$.

Corollary 5.8. Let $M_{\text {reg }}=\bar{M}_{r e g} \backslash D$, then $\omega_{1}$ is smooth on $M_{r e g} .\left(M_{1}, d_{1}\right)$ is isometric to $\overline{\left(M_{r e g}, \omega_{1}\right)}$. Proof. Note that $M_{\text {reg }} \backslash(\bar{M} \backslash \bar{D})=M_{\text {reg }} \cap \bar{D}$ has real codimension larger than 2 in $\left(M_{\text {reg }}, \omega_{1}\right)$. So $\bar{M} \backslash \bar{D}$ is dense in $M_{\text {reg }}$. We conclude

$$
\left(M_{1}, d_{1}\right)=\overline{\left(\bar{M} \backslash \bar{D}, \omega_{1}\right)}=\overline{\left(M_{r e g}, \omega_{1}\right)} .
$$

Proposition 5.9. $M_{\text {reg }}=\mathcal{R}$, the regular set of $M_{1}$.

Proof. Since $M_{\text {reg }}$ has smooth structure in $M_{1}$, we have $M_{\text {reg }} \subset \mathcal{R}$. Next we show the converse. We argue by contradiction. Suppose $p \in \mathcal{R} \backslash M_{\text {reg }}$, then there exists a family of points $p_{l} \in M_{\text {sing }}$ such that $p_{l} \stackrel{d_{G H}}{\longrightarrow} p$, where $M_{\text {sing }}=\left(\Phi^{-1}\left(\Phi(\bar{M})_{\text {sing }}\right)\right) \backslash D$. By $C^{1, \alpha}$ convergence on $\mathcal{R}$, there exist $C, r>0$ independent of $l$ and a sequence of harmonic coordinates on $B_{\omega_{l}}\left(p_{l}, r\right)$ such that $C^{-1} \omega_{E} \leq \omega_{l} \leq C \omega_{E}$ where $\omega_{E}$ is the Euclidean metric in this coordinate. Furthermore, the sequence of harmonic coordinate can be perturbed to a holomorphic coordinate on $B_{\omega_{l}}\left(p_{l}, r\right)$ [24]. Denote $m=\operatorname{dim}_{\mathbb{C}}\left(M_{\text {sing }}\right)$. Then

$$
\begin{gathered}
\operatorname{Vol}_{\omega_{l}}\left(M_{\text {sing }} \cap B_{\omega_{l}}\left(p_{l}, r\right)\right)=\int_{M_{\text {sing }} \cap B_{\omega_{l}}\left(p_{l}, r\right)} \omega_{l}^{m} \\
\geq \int_{M_{\text {sing }} \cap B_{\omega_{E}}\left(C^{-\frac{1}{2}} r\right)}\left(C^{-1} \omega_{E}\right)^{m}
\end{gathered}
$$


which has a uniform lower bound. However, this contradicts with the degeneration of the limit metric $\eta_{1}$ along $M_{\text {sing }}$ :

$$
\begin{aligned}
\operatorname{Vol}_{\omega_{l}} & \left(M_{\text {sing }} \cap B_{\omega_{l}}\left(p_{l}, r\right)\right) \leq \operatorname{Vol}_{\omega_{l}}\left(M_{\text {sing }}\right) \\
= & \int_{M_{\text {sing }}} \omega_{l}^{m}=\int_{M_{\text {sing }}}\left((1-l) \omega_{0}+l \eta_{1}\right)^{m}
\end{aligned}
$$

which tends to 0 as $l \rightarrow 1$, where the last equality bases on a Lemma ([16] P410). So we have $M_{\text {reg }} \supset \mathcal{R}$.

5.2. $L^{\infty}$ estimate and gradient estimate to holomorphic sections. In this subsection we obtain the $L^{\infty}$ estimate and gradient estimate to holomorphic section $s \in H^{0}\left(\mathcal{R}, k\left(K_{\bar{M}}+D\right)\right) . h=\omega_{1}^{-n k}$ is chosen as the Hermitian metric of line bundle $k\left(K_{\bar{M}}+D\right)$, where $k \in \mathbb{Z}$. The curvature form $\Theta_{h}$ of Hermitian metric $h=\omega_{1}^{-n k}$ is $k \omega_{1}$. By Lemma 2.4, we have the following formulas.

Lemma 5.10. For $s \in H^{0}\left(\mathcal{R}, k\left(K_{\bar{M}}+D\right)\right)$, there exists a constant $C$ such that

$$
\triangle_{\omega_{1}}|s|^{2}=|\nabla s|^{2}-k n|s|^{2}
$$

and

$$
\triangle_{\omega_{1}}|\nabla s|^{2} \geq|\bar{\nabla} \nabla s|^{2}+|\nabla \nabla s|^{2}-C k|\nabla s|^{2}-k \nabla_{j}\left(\omega_{1}\right)_{i \bar{j}}\left\langle s, \nabla_{\bar{i}} \bar{s}\right\rangle .
$$

Proof. Since on $\mathcal{R}, \operatorname{Ric}\left(\omega_{1}\right)=-\omega_{1}$. So these formulas are directly derived from Lemma 2.4,

In order to applying Moser iteration, the Sobolev inequality on $\mathcal{R}$ is needed. The following two Lemmas are due to Song (Lemma 3.7 and 4.6 [21]).

Lemma 5.11. There is a family of cut-off functions $\rho_{\epsilon} \in C_{0}^{\infty}(\mathcal{R})$ with $0<\rho_{\epsilon}<1$ such that $\rho_{\epsilon}^{-1}(1)$ forms an exhaustion of $\mathcal{R}$ and

$$
\int_{\mathcal{R}}\left|\nabla \rho_{\epsilon}\right|^{2} \omega_{1}^{n} \rightarrow 0
$$

Lemma 5.12. Fix any $0<r<R$, the Sobolev constant on $B_{\omega_{l}}(x, r)$ is uniformly bounded by a constant $C_{S}$ depending on upper bound of $R, R^{-1}$ and $(R-r)^{-1}$. More precisely, for any $l \in\left[\frac{1}{2}, 1\right)$ and $f \in C_{0}^{1}\left(B_{\omega_{l}}(x, r)\right)$,

$$
C_{S}\left(\int_{B_{\omega_{l}}(x, r)}|f|^{\frac{2 n}{n-1}} \omega_{l}^{n}\right)^{\frac{n-1}{n}} \leq \int_{B_{\omega_{l}}(x, r)}\left(|f|^{2}+|\nabla f|^{2}\right) \omega_{l}^{n} .
$$

Fix $0<r<R$ such that $B_{\omega_{1}}(x, r) \subset B_{\omega_{1}}(x, 2 r) \subset B_{\omega_{1}}(x, R)$.

Lemma 5.13. If $f \in C_{0}^{1}\left(B_{\omega_{1}}(x, r) \cap \mathcal{R}\right)$, then there exists a constant $C$ depending on $R, R^{-1}$ and $(R-r)^{-1}$ such that

$$
C\left(\int_{B_{\omega_{1}}(x, r) \cap \mathcal{R}}|f|^{\frac{2 n}{n-1}} \omega_{1}^{n}\right)^{\frac{n-1}{n}} \leq \int_{B_{\omega_{1}}(x, r) \cap \mathcal{R}}\left(|f|^{2}+|\nabla f|^{2}\right) \omega_{1}^{n} .
$$

Proof. Let $f_{\epsilon}=\rho_{\epsilon} f$, where $\rho_{\epsilon}$ is constructed as Lemma 5.11 and $\Omega_{\epsilon}=\operatorname{Supp} f_{\epsilon}$. Then $\omega_{l}$ uniformly converge to $\omega_{1}$ on $\Omega_{\epsilon}$ as $l$ tends to 1 for a fixed $\epsilon$. Therefore $\Omega_{\epsilon} \subset B_{\omega_{l}}(x, r)$ for $l$ sufficiently close to 1. By Lemma [5.12, we have

$$
C_{S}\left(\int_{B_{\omega_{l}}(x, r)}\left|f_{\epsilon}\right|^{\frac{2 n}{n-1}} \omega_{l}^{n}\right)^{\frac{n-1}{n}} \leq \int_{B_{\omega_{l}}(x, r)}\left(\left|f_{\epsilon}\right|^{2}+\left|\nabla f_{\epsilon}\right|^{2}\right) \omega_{l}^{n} .
$$


Let $l \rightarrow 1$, the above inequality gives

$$
C_{S}\left(\int_{B_{\omega_{1}}(x, r)}\left|f_{\epsilon}\right|^{\frac{2 n}{n-1}} \omega_{1}^{n}\right)^{\frac{n-1}{n}} \leq \int_{B_{\omega_{1}}(x, r)}\left(\left|f_{\epsilon}\right|^{2}+\left|\nabla f_{\epsilon}\right|^{2}\right) \omega_{1}^{n} .
$$

Note that by letting $\epsilon \rightarrow 0$, we get

$$
\int_{B_{\omega_{1}}(x, r)}\left|f_{\epsilon}\right|^{\frac{2 n}{n-1}} \omega_{1}^{n} \rightarrow \int_{B_{\omega_{1}}(x, r)}|f|^{\frac{2 n}{n-1}} \omega_{1}^{n}
$$

and

$$
\int_{B_{\omega_{1}}(x, r)}\left|f_{\epsilon}\right|^{2} \omega_{1}^{n} \rightarrow \int_{B_{\omega_{1}}(x, r)}|f|^{2} \omega_{1}^{n}
$$

By some calculations we have

$$
\left.\left|\int_{B_{\omega_{1}}(x, r)}\right| \nabla f_{\epsilon}\right|^{2} \omega_{1}^{n}-\int_{B_{\omega_{1}}(x, r)}|\nabla f|^{2} \omega_{1}^{n}|=| \int_{B_{\omega_{1}(x, r)}}\left(\left|\nabla \rho_{\epsilon}\right|^{2}|f|^{2}+\left(\left|\rho_{\epsilon}\right|^{2}|\nabla f|^{2}-|\nabla f|^{2}\right)\right) \omega_{1}^{n} \mid
$$

which tends to 0 . So this Lemma is proved.

Lemma 5.14. There exists a constant $C$ independent of $k$ such that if $s \in H^{0}\left(\mathcal{R}, k\left(K_{\bar{M}}+D\right)\right)$, then

$$
\int_{B_{\omega_{1}}\left(x, \frac{7}{4} r\right) \cap \mathcal{R}}|\nabla s|^{2} \omega_{1}^{n} \leq C k r^{-2} \int_{B_{\omega_{1}}(x, 2 r) \cap \mathcal{R}}|s|^{2} \omega_{1}^{n}
$$

and

$$
\int_{B_{\omega_{1}}\left(x, \frac{7}{4} r\right) \cap \mathcal{R}}\left(|\bar{\nabla} \nabla s|^{2}+|\nabla \nabla s|^{2}\right) \omega_{1}^{n} \leq C k^{2} r^{-4} \int_{B_{\omega_{1}}(x, 2 r) \cap \mathcal{R}}|s|^{2} \omega_{1}^{n} .
$$

Proof. Let $\vartheta \in C_{0}^{\infty}\left(B_{\omega_{1}}\left(x, \frac{15}{8} r\right) \cap \mathcal{R}\right)$ be any cut-off function such that $0 \leq \vartheta \leq 1,|\nabla \vartheta| \leq 10 r^{-2}$ and $\vartheta=1$ on $B_{\omega_{1}}\left(x, \frac{7}{4} r\right) \cap \mathcal{R}$, then by Bochner formula we have

$$
\int_{\mathcal{R}} \vartheta^{2}|\nabla s|^{2} \omega_{1}^{n}=n k \int_{\mathcal{R}} \vartheta^{2}|s|^{2} \omega_{1}^{n}+\int_{\mathcal{R}} \vartheta^{2} \triangle|s|^{2} \omega_{1}^{n}
$$

Note that

$$
\int_{\mathcal{R}} \vartheta^{2} \triangle|s|^{2} \omega_{1}^{n}=-2 \int_{\mathcal{R}} \vartheta \nabla_{\bar{i}} \vartheta\left\langle\nabla_{i} s, \bar{s}\right\rangle \omega_{1}^{n} \leq \frac{1}{2} \int_{\mathcal{R}} \vartheta^{2}|\nabla s|^{2} \omega_{1}^{n}+2 \int_{\mathcal{R}}|\nabla \vartheta|^{2}|s|^{2} \omega_{1}^{n} .
$$

Therefore,

$$
\int_{B_{\omega_{1}}\left(x, \frac{7}{4} r\right) \cap \mathcal{R}}|\nabla s|^{2} \omega_{1}^{n} \leq C k r^{-2} \int_{B_{\omega_{1}}(x, 2 r) \cap \mathcal{R}}|s|^{2} \omega_{1}^{n} .
$$

For the second inequality, also by the Bochner formula

$$
\int_{\mathcal{R}} \vartheta^{2}\left(|\bar{\nabla} \nabla s|^{2}+|\nabla \nabla s|^{2}\right) \omega_{1}^{n} \leq \int_{\mathcal{R}} \vartheta^{2}\left(\triangle|\nabla s|^{2}+C k|\nabla s|^{2}+k \nabla_{j}\left(\omega_{1}\right)_{i \bar{j}}\left\langle s, \nabla_{\bar{i}} \bar{s}\right\rangle\right) \omega_{1}^{n} .
$$

Note that

$$
\int_{\mathcal{R}} \vartheta^{2} \triangle|\nabla s|^{2} \omega_{1}^{n}=-2 \int_{\mathcal{R}} \vartheta \nabla_{i} \vartheta \nabla_{\bar{i}}|\nabla s|^{2} \omega_{1}^{n} \leq \frac{1}{4} \int_{\mathcal{R}} \vartheta^{2}\left(|\bar{\nabla} \nabla s|^{2}+|\nabla \nabla s|^{2}\right) \omega_{1}^{n}+C \int_{\mathcal{R}}|\nabla \vartheta|^{2}+|\nabla s|^{2} \omega_{1}^{n}
$$


and

$$
\begin{aligned}
\int_{\mathcal{R}} k \vartheta^{2} \nabla_{j}\left(\omega_{1}\right)_{i j}\left\langle s, \nabla_{\bar{i}} \bar{s}\right\rangle \omega_{1}^{n}= & -k \int_{\mathcal{R}} \vartheta^{2}\left(\omega_{1}\right)_{i \bar{j}}\left(\left\langle\nabla_{j} s, \nabla_{\bar{i}} \bar{s}\right\rangle+\left\langle s, \nabla_{\bar{j}} \nabla_{\bar{i}} \bar{s}\right\rangle\right) \omega_{1}^{n}-2 k \int_{\mathcal{R}} \vartheta \nabla_{j} \vartheta\left(\omega_{1}\right)_{i \bar{j}}\left\langle s, \nabla_{\bar{i}} \bar{s}\right\rangle \omega_{1}^{n} \\
\leq & \frac{1}{4} \int_{\mathcal{R}} \vartheta^{2}\left(|\bar{\nabla} \nabla s|^{2}+|\nabla \nabla s|^{2}\right) \omega_{1}^{n}+C k \int_{\mathcal{R}} \vartheta^{2}|s|^{2} \omega_{1}^{n}+C k \int_{\mathcal{R}} \vartheta^{2}|\nabla s|^{2} \omega_{1}^{n} \\
& +C k \int_{\mathcal{R}}|\nabla \vartheta|^{2}|\nabla s|^{2} \omega_{1}^{n}
\end{aligned}
$$

Summing up these estimates we have

$$
\int_{B_{\omega_{1}}\left(x, \frac{7}{4} r\right) \cap \mathcal{R}}\left(|\bar{\nabla} \nabla s|^{2}+|\nabla \nabla s|^{2}\right) \omega_{1}^{n} \leq C k r^{-2} \int_{B_{\omega_{1}}(x, 2 r) \cap \mathcal{R}}|\nabla s|^{2} \omega_{1}^{n}+C k \int_{B_{\omega_{1}}(x, 2 r) \cap \mathcal{R}}|s|^{2} \omega_{1}^{n} .
$$

Applying the first inequality we obtain the second estimate.

Proposition 5.15. There exists a constant $C(R, r)$ independent of $k$ such that if $s \in H^{0}\left(\mathcal{R}, k\left(K_{\bar{M}}+\right.\right.$ $D)$ ), then

$$
\sup _{B_{\omega_{1}}(x, r) \cap \mathcal{R}}|s|^{2} \leq C(R, r) k^{n} r^{-2 n} \int_{B_{\omega_{1}}(x, 2 r) \cap \mathcal{R}}|s|^{2} \omega_{1}^{n}
$$

and

$$
\sup _{B_{\omega_{1}}(x, r) \cap \mathcal{R}}|\nabla s|^{2} \leq C(R, r) k^{n+1} r^{-2 n-2} \int_{B_{\omega_{1}}(x, 2 r) \cap \mathcal{R}}|s|^{2} \omega_{1}^{n} .
$$

Proof. Choose a cut-off function $\vartheta \in C_{0}^{\infty}\left(B_{\omega_{1}}(x, 2 r) \cap \mathcal{R}\right)$. Then for any $p \geq \frac{n}{n-1}$, by Lemma [5.10, we have

$$
\begin{aligned}
\left.\left.\int_{\mathcal{R}} \vartheta^{2}|\nabla| s\right|^{p}\right|^{2} \omega_{1}^{n}= & \frac{p^{2}}{4(p-1)} \int_{\mathcal{R}} \vartheta^{2} \nabla_{i}|s|^{2(p-1)} \nabla_{\bar{i}}|s|^{2} \omega_{1}^{n} \\
= & \frac{p^{2}}{4(p-1)} \int_{\mathcal{R}}\left(-\vartheta^{2}|s|^{2(p-1)} \triangle_{\omega_{1}}|s|^{2}-2 \vartheta \cdot \nabla_{i} \vartheta \cdot|s|^{2(p-1)} \cdot \nabla_{\bar{i}}|s|^{2}\right) \omega_{1}^{n} \\
\leq & \frac{p^{2}}{4(p-1)} \int_{\mathcal{R}}-\vartheta^{2}|s|^{2(p-1)}|\nabla s|^{2} \omega_{1}^{n}+n k \frac{p^{2}}{4(p-1)} \int_{\mathcal{R}} \vartheta^{2}|s|^{2 p} \omega_{1}^{n} \\
& \quad+\frac{p^{2}}{4(p-1)} \int_{\mathcal{R}} \vartheta \cdot|\nabla \vartheta| \cdot|s|^{2(p-1)} \cdot|\nabla s| \cdot|s| \omega_{1}^{n} .
\end{aligned}
$$

By Cauchy-Schwarz inequality,

$$
\int_{\mathcal{R}} \vartheta \cdot|\nabla \vartheta| \cdot|s|^{2(p-1)} \cdot|\nabla s| \cdot|s| \omega_{1}^{n} \leq \int_{\mathcal{R}} \vartheta^{2}|s|^{2(p-1)}|\nabla s|^{2} \omega_{1}^{n}+\frac{1}{4} \int_{\mathcal{R}}|\nabla \vartheta|^{2}|s|^{2 p} \omega_{1}^{n} .
$$

Therefore

$$
\left.\left.\int_{\mathcal{R}} \vartheta^{2}|\nabla| s\right|^{p}\right|^{2} \omega_{1}^{n} \leq C p k \int_{\mathcal{R}}\left(\vartheta^{2}+|\nabla \vartheta|^{2}\right)|s|^{2 p} \omega_{1}^{n} .
$$

By Lemma 5.13

$$
\left(\int_{\mathcal{R}}\left(\vartheta|s|^{p}\right)^{\frac{2 n}{n-1}} \omega_{1}^{n}\right)^{\frac{n-1}{n}} \leq C p k \int_{\mathcal{R}}\left(\vartheta^{2}+|\nabla \vartheta|^{2}\right)|s|^{2 p} \omega_{1}^{n} .
$$


Put $p_{j}=\nu^{j+1}$ for $j \geq 0$, where $\nu=\frac{n}{n-1}$. Define a family of radius inductively by $r_{0}=\frac{3}{2} r$ and $r_{j}=r_{j-1}-2^{-j-1} r . \quad B_{j}$ is denoted by $B_{\omega_{1}}\left(x, r_{j}\right) \cap \mathcal{R}$. We choose a family of cut-off functions $\vartheta_{j} \in C_{0}^{\infty}\left(B_{j}\right)$ such that

$$
0 \leq \vartheta_{j} \leq 1,\left|\nabla \vartheta_{j}\right| \leq 2^{j+2} r^{-1} \text { and } \vartheta_{j}=1 \text { on } B_{j+1} .
$$

Thus (5.2) gives, by setting $\vartheta=\vartheta_{j}$

$$
\left(\int_{B_{j+1}}|s|^{2 p_{j+1}} \omega_{1}^{n}\right)^{\frac{1}{p_{j+1}}} \leq\left(C p_{j} k\right)^{\frac{1}{p_{j}}} 4^{\frac{j}{p_{j}}} r^{-\frac{2}{p_{j}}}\left(\int_{B_{j}}|s|^{2 p_{j}} \omega_{1}^{n}\right)^{\frac{1}{p_{j}}} .
$$

By the iteration argument, we see

$$
\sup _{B_{\omega_{1}}(x, r) \cap \mathcal{R}}|s|^{2} \leq C k^{n-1} r^{-2(n-1)}\left(\int_{B_{0}}|s|^{\frac{2 n}{n-1}} \omega_{1}^{n}\right)^{\frac{n}{n-1}} \leq C k^{n-1} r^{-2(n-1)} \int_{B_{0}}\left(|s|^{2}+|\nabla s|^{2}\right) \omega_{1}^{n} .
$$

According to Lemma 5.14, we get the first estimate.

Next we prove the second inequality. Let $\vartheta$ and $p$ as above. By Lemma 5.10, we have

$$
\begin{aligned}
\left.\left.\int_{\mathcal{R}} \vartheta^{2}|\nabla| \nabla S\right|^{p}\right|^{2} \omega_{1}^{n}= & \frac{p^{2}}{4(p-1)} \int_{\mathcal{R}} \vartheta^{2} \cdot \nabla_{i}|\nabla s|^{2(p-1)} \cdot \nabla_{\bar{i}}|\nabla s|^{2} \omega_{1}^{n} \\
= & \frac{p^{2}}{4(p-1)} \int_{\mathcal{R}}\left(-\vartheta^{2}|\nabla s|^{2(p-1)} \triangle|\nabla s|^{2}-2 \vartheta \cdot \nabla_{i} \vartheta \cdot|\nabla s|^{2(p-1)} \nabla_{\bar{i}}|\nabla s|^{2}\right) \omega_{1}^{n} \\
\leq & \frac{p^{2}}{4(p-1)} \int_{\mathcal{R}}\left(-\vartheta^{2}|\nabla s|^{2(p-1)}\left(|\bar{\nabla} \nabla s|^{2}+|\nabla \nabla s|^{2}\right)+k \nabla_{j}\left(\omega_{1}\right)_{i \bar{j}}\left\langle s, \nabla_{\bar{i}} \bar{s}\right\rangle \cdot \vartheta^{2}|\nabla s|^{2(p-1)}\right. \\
& \left.\quad+C k \vartheta^{2}|\nabla s|^{2 p}-2 \vartheta \cdot \nabla_{i} \vartheta \cdot|\nabla s|^{2(p-1)} \cdot \nabla_{\bar{i}}|\nabla s|^{2}\right) \omega_{1}^{n}
\end{aligned}
$$

The term $\int_{\mathcal{R}} k \nabla_{j}\left(\omega_{1}\right)_{i j}\left\langle s, \nabla_{\bar{i}} \bar{s}\right\rangle \cdot \vartheta^{2}|\nabla s|^{2(p-1)} \omega_{1}^{n}$ can be estimate by integration by parts as follows

$$
\begin{aligned}
& \int_{\mathcal{R}} k \nabla_{j}\left(\omega_{1}\right)_{i \bar{j}}\left\langle s, \nabla_{\bar{i}} \bar{s}\right\rangle \cdot \vartheta^{2}|\nabla s|^{2(p-1)} \omega_{1}^{n} \\
= & -k \int_{\mathcal{R}}\left(\omega_{1}\right)_{i \bar{j}}\left(\vartheta^{2}|\nabla s|^{2(p-1)}\left(\left\langle\nabla_{j} s, \nabla_{\bar{i}} \bar{s}\right\rangle+\left\langle s, \nabla_{j} \nabla_{\bar{i}} \bar{s}\right\rangle\right)+(p-1) \vartheta^{2}|\nabla s|^{2(p-2)} \nabla_{\bar{j}}|\nabla s|^{2}\left\langle s, \nabla_{\bar{i}} \bar{s}\right\rangle\right. \\
& \left.+2 \vartheta \nabla_{j} \vartheta|\nabla s|^{2(p-1)}\left\langle s, \nabla_{\bar{i}} \bar{s}\right\rangle\right) \\
\leq & \frac{1}{2} \int_{\mathcal{R}} \vartheta^{2}|\nabla s|^{2(p-1)}\left(|\bar{\nabla} \nabla s|^{2}+|\nabla \nabla s|^{2}\right) \omega_{1}^{n}+C(p-1)^{2} k^{2} \int_{\mathcal{R}} \vartheta^{2}|s|^{2}|\nabla s|^{2(p-1)} \omega_{1}^{n} \\
& +C k \int_{\mathcal{R}}\left(|\nabla \vartheta|^{2}|s|^{2}|\nabla s|^{2(p-1)}+\vartheta^{2}|\nabla s|^{2 p}\right) \omega_{1}^{n} .
\end{aligned}
$$

Note that

$-2 \int_{\mathcal{R}} \vartheta \cdot \nabla_{i} \vartheta \cdot|\nabla s|^{2(p-1)} \cdot \nabla_{\bar{i}}|\nabla s|^{2} \omega_{1}^{n} \leq \frac{1}{2} \int_{\mathcal{R}} \vartheta^{2}|\nabla s|^{2(p-1)}\left(|\bar{\nabla} \nabla s|^{2}+|\nabla \nabla s|^{2}\right) \omega_{1}^{n}+C \int_{\mathcal{R}}|\nabla \vartheta|^{2}|\nabla s|^{2 p} \omega_{1}^{n}$.

Summing up these estimates we conclude

$\left.\left.\int_{\mathcal{R}} \vartheta^{2}|\nabla| \nabla S\right|^{p}\right|^{2} \omega_{1}^{n} \leq C p^{3} k \int_{\mathcal{R}}\left(k \vartheta^{2}|\nabla s|^{2(p-1)}|s|^{2}+|\nabla \vartheta|^{2}|s|^{2}|\nabla s|^{2(p-1)}+\vartheta^{2}|\nabla s|^{2 p}+|\nabla s|^{2 p}|\nabla \vartheta|^{2}\right) \omega_{1}^{n}$.

Applying the Lemma 5.13 


$$
\begin{aligned}
\left(\int_{\mathcal{R}}\left(\vartheta|\nabla s|^{p}\right)^{\frac{2 n}{n-1}} \omega_{1}^{n}\right)^{\frac{n-1}{n}} & \leq C p^{3} k \int_{\mathcal{R}}\left(k \vartheta^{2}|\nabla s|^{2(p-1)}|s|^{2}+|\nabla \vartheta|^{2}|s|^{2}|\nabla s|^{2(p-1)}\right. \\
& \left.+\vartheta^{2}|\nabla s|^{2 p}+|\nabla s|^{2 p}|\nabla \vartheta|^{2}\right) \omega_{1}^{n} .
\end{aligned}
$$

Put $p_{j}=\nu^{j+1}$ for $j \geq 0$, where $\nu=\frac{n}{n-1}$. Define a family of radius inductively by $r_{0}=\frac{3}{2} r$ and $r_{j}=r_{j-1}-2^{-j-1} r$. $B_{j}$ is denoted by $B_{\omega_{1}}\left(x, r_{j}\right) \cap \mathcal{R}$. We choose a family of cut-off functions $\vartheta_{j} \in C_{0}^{\infty}\left(B_{j}\right)$ such that

$$
0 \leq \vartheta_{j} \leq 1,\left|\nabla \vartheta_{j}\right| \leq 2^{j+2} r^{-1} \text { and } \vartheta_{j}=1 \text { on } B_{j+1} .
$$

By setting $\vartheta=\vartheta_{j}$, the above inequality gives

$$
\left(\int_{B_{j+1}}|\nabla s|^{2 p_{j+1}} \omega_{1}^{n}\right)^{\frac{n}{n-1}} \leq C p_{j}^{3} k 4^{j} r^{-2} \int_{B_{j}}\left(|\nabla s|^{2 p_{j}}+k|\nabla s|^{2\left(p_{j}-1\right)}|s|^{2}\right) \omega_{1}^{n} .
$$

Case 1: If $\left(\int_{B_{j}}|\nabla s|^{2 p_{j}} \omega_{1}^{n}\right)^{\frac{1}{p_{j}}} \geq k\left(\int_{B_{j}}|s|^{2 p_{j}} \omega_{1}^{n}\right)^{\frac{1}{p_{j}}}$ for all $j \geq 0$. Then

$$
\int_{B_{j}} k|\nabla s|^{2\left(p_{j}-1\right)}|s|^{2} \omega_{1}^{n} \leq k\left(\int_{B_{j}}|\nabla s|^{2 p_{j}} \omega_{1}^{n}\right)^{\frac{p_{j}-1}{p_{j}}}\left(\int_{B_{j}}|s|^{2 p_{j}} \omega_{1}^{n}\right)^{\frac{1}{p_{j}}} \leq \int_{B_{j}}|\nabla s|^{2 p_{j}} \omega_{1}^{n} .
$$

Then (5.17) gives

$$
\left(\int_{B_{j+1}}|\nabla s|^{2 p_{j+1}} \omega_{1}^{n}\right)^{\frac{1}{p_{j+1}}} \leq C\left(k r^{-2}\right)^{\frac{1}{p_{j}}} 4^{\frac{j}{p_{j}}} p_{j}^{\frac{3}{p_{j}}}\left(\int_{B_{j}}|\nabla s|^{2 p_{j}} \omega_{1}^{n}\right)^{\frac{1}{p_{j}}}
$$

By iteration argument we get

$$
\sup _{B_{\omega_{1}}(x, r) \cap \mathcal{R}}|\nabla s|^{2} \leq C\left(k r^{-2}\right)^{n-1}\left(\int_{B_{0}}|\nabla s|^{\frac{2 n}{n-1}} \omega_{1}^{n}\right)^{\frac{n-1}{n}} .
$$

By Lemma 5.13 and a cut-off argument, we have

$$
\left(\int_{B_{0}}|\nabla s|^{\frac{2 n}{n-1}} \omega_{1}^{n}\right)^{\frac{n-1}{n}} \leq C \int_{B_{\omega_{1}}\left(x, \frac{7}{4} r\right) \cap \mathcal{R}}\left(|\bar{\nabla} \nabla s|^{2}+|\nabla \nabla s|^{2}+r^{-2}|\nabla s|^{2}\right) \omega_{1}^{n}
$$

According to Lemma 5.14 we get

$$
\sup _{B_{\omega_{1}}(x, r) \cap \mathcal{R}}|\nabla s|^{2} \leq C\left(k r^{-2}\right)^{n+1} \int_{B_{\omega_{1}}(x, 2 r) \cap \mathcal{R}}|s|^{2} \omega_{1}^{n} .
$$

Case2: There exists $j_{0}$ such that $\left(\int_{B_{j}}|\nabla s|^{2 p_{j}} \omega_{1}^{n}\right)^{\frac{1}{p_{j}}} \geq k\left(\int_{B_{j}}|s|^{2 p_{j}} \omega_{1}^{n}\right)^{\frac{1}{p_{j}}}$ for all $j>j_{0}$, but

$$
\left(\int_{B_{j_{0}}}|\nabla s|^{2 p_{j_{0}}} \omega_{1}^{n}\right)^{\frac{1}{p_{j_{0}}}}<k\left(\int_{B_{j_{0}}}|s|^{2 p_{j_{0}}} \omega_{1}^{n}\right)^{\frac{1}{p_{j_{0}}}} .
$$

Then

$$
\int_{B_{j_{0}}} k|\nabla s|^{2\left(p_{j_{0}}-1\right)}|s|^{2} \omega_{1}^{n} \leq k\left(\int_{B_{j_{0}}}|\nabla s|^{2 p_{j_{0}}} \omega_{1}^{n}\right)^{\frac{p_{j_{0}}-1}{p_{j_{0}}}}\left(\int_{B_{j_{0}}}|s|^{2 p_{j_{0}}} \omega_{1}^{n}\right)^{\frac{1}{p_{j_{0}}}} \leq k^{p_{j_{0}}} \int_{B_{j_{0}}}|s|^{2 p_{j_{0}}} \omega_{1}^{n} .
$$


By the iteration argument and (5.17), we have

$$
\sup _{B_{\omega_{1}}(x, r) \cap \mathcal{R}}|\nabla s|^{2} \leq C k\left(k r^{-2}\right)^{\frac{n}{p_{j_{0}}}}\left(\int_{B_{j_{0}}}|s|^{2 p_{j_{0}}} \omega_{1}^{n}\right)^{\frac{1}{p_{j_{0}}}} .
$$

The supermum of $|\nabla s|$ follows from

$$
\left(\int_{B_{j_{0}}}|s|^{2 p_{j_{0}}} \omega_{1}^{n}\right)^{\frac{1}{p_{j_{0}}}} \leq\left(\sup _{B_{j_{0}}}|s|\right)^{\frac{2 p_{j_{0}}-2}{p_{j_{0}}}}\left(\int_{B_{j_{0}}}|s|^{2} \omega_{1}^{n}\right)^{\frac{1}{p_{j_{0}}}} \leq C\left(k r^{-2}\right)^{n-\frac{n}{p_{j_{0}}}} \int_{B_{j_{0}}}|s|^{2} \omega_{1}^{n} .
$$

Case 3: If $\left(\int_{B_{j}}|\nabla s|^{2 p_{j}} \omega_{1}^{n}\right)^{\frac{1}{p_{j}}} \leq k\left(\int_{B_{j}}|s|^{2 p_{j}} \omega_{1}^{n}\right)^{\frac{1}{p_{j}}}$ for infinite $i$, then

$$
\sup _{B_{\omega_{1}}(x, r) \cap \mathcal{R}}|\nabla s|^{2} \leq k \sup _{B_{\omega_{1}}(x, r) \cap \mathcal{R}}|s| \leq C k^{n+1} r^{-2 n} \int_{B_{\omega_{1}}(x, 2 r) \cap \mathcal{R}}|s|^{2} \omega_{1}^{n} .
$$

5.3. $L^{2}$ estimate. In order to construct global holomorphic section on line bundle $k\left(K_{\bar{M}}+D\right)$, we need the following version of $L^{2}$-estimate due to Demailly (Theorem 5.1 [12]).

Theorem 5.18. Let $(M, \omega)$ be a n-dimensional complete Kähler manifold and $L$ be a holomorphic line bundle over $M$ equipped with a smooth hermitian metric such that $\Theta_{h} \geq \delta \omega$. Then for every L-value $(n, 1)$ form $\tau$ satisfying

$$
\bar{\partial} \tau=0, \quad \int_{M}|\tau|_{h, \omega}^{2} \omega^{n}<\infty,
$$

there exists a $L$-valued $(n, 0)$ form $u$ such that $\bar{\partial} u=\tau$ and

$$
\int_{M}|u|_{h, \omega}^{2} \omega^{n} \leq \frac{1}{\delta} \int_{M}|\tau|_{h, \omega}^{2} \omega^{n}
$$

For the singular hermitian metric $h$ on $L$, by the approximation argument, we have

Corollary 5.19. Let $(M, \omega)$ be a n-dimensional complete Kähler manifold and $L$ be a holomorphic line bundle over $M$ equipped with a singular hermitian metric such that $\Theta_{h} \geq \delta \omega$ in the current sense. Then for every $L$-value $(n, 1)$ form $\tau$ satisfying

$$
\bar{\partial} \tau=0, \quad \int_{M}|\tau|_{h, \omega}^{2} \omega^{n}<\infty,
$$

there exists a L-valued $(n, 0)$ form $u$ such that $\bar{\partial} u=\tau$ and

$$
\int_{M}|u|_{h, \omega}^{2} \omega^{n} \leq \frac{1}{\delta} \int_{M}|\tau|_{h, \omega}^{2} \omega^{n}
$$

Proposition 5.20. $\left(\mathcal{R}=M_{\text {reg }}, k \omega_{1}\right)$ is a Kähler manifold (not complete). $k\left(K_{\bar{M}}+D\right)$ is a holomorphic line bundle over $\mathcal{R}$. Choosing a hermitian metric $h=\omega_{1}^{-n k}$, then the curvature form $\Theta_{h}=k \omega_{1}$. For any smooth $k\left(K_{\bar{M}}+D\right)$-valued $(0,1)$ form $\tau$ satisfying

$$
\bar{\partial} \tau=0, \quad \operatorname{Supp} \tau \subset \mathcal{R}
$$

there exists a $k\left(K_{\bar{M}}+D\right)$-valued section $\varsigma$ such that $\bar{\partial} \varsigma=\tau$ and

$$
\int_{\mathcal{R}}|\varsigma|_{h}^{2}\left(k \omega_{1}\right)^{n} \leq \int_{\mathcal{R}}|\tau|_{h, k \omega_{1}}^{2}\left(k \omega_{1}\right)^{n} .
$$


Proof. Since $K_{\bar{M}}+D$ is big and semi-ample over $\bar{M}$, by Lemma 4.1, there exists an effective divisor $E$ on $\bar{M}$ such that $K_{\bar{M}}+D-\epsilon E$ is ample for all sufficiently small $\epsilon>0$. Let $s_{E}$ be the defining section of $E$ and $h_{E}$ be a smooth hermitian metric satisfying $\eta_{1}-\epsilon \Theta_{E}>0$, where $\eta_{1}$ is constructed as section 3 and $\Theta_{E}$ is the curvature form. We consider the following Monge-Ampère equation

$$
\left(\eta_{1}-\epsilon \Theta_{E}-\sqrt{-1} \partial \bar{\partial} \log \log ^{2}\left|s_{D}\right|^{2}+\sqrt{-1} \partial \bar{\partial} u_{1, \epsilon}\right)^{n}=e^{u_{1, \epsilon}} \cdot \frac{\Omega}{\left|s_{D}\right|^{2} \log ^{2}\left|s_{D}\right|^{2}} .
$$

Fixed a small $\alpha>0$, this equation is rewritten as

$$
\left((1-\alpha) \eta_{1}+\alpha\left(\eta_{1}-\frac{\epsilon}{\alpha} \Theta_{E}\right)-\sqrt{-1} \partial \bar{\partial} \log \log ^{2}\left|s_{D}\right|^{2}+\sqrt{-1} \partial \bar{\partial} u_{1, \epsilon}\right)^{n}=e^{u_{1, \epsilon}} \cdot \frac{\Omega}{\left|s_{D}\right|^{2} \log ^{2}\left|s_{D}\right|^{2}} .
$$

By the same argument of subsection 5.5, we know that $\omega_{1, \epsilon}=\eta_{1}-\epsilon \Theta_{E}-\sqrt{-1} \partial \bar{\partial} \log \log ^{2}\left|s_{D}\right|^{2}+$ $\sqrt{-1} \partial \bar{\partial} u_{1, \epsilon} C_{l o c}^{\infty}\left(M_{r e g}\right)$-converge to $\omega_{1}$ as $\epsilon$ tends to 0 . Now we define a family of hermitian metric

$$
h_{\epsilon}=e^{-k u_{1, \epsilon}}\left(\frac{\Omega}{\left|s_{D}\right|^{2} \log ^{2}\left|s_{D}\right|^{2}}\right)^{-k} e^{-\epsilon k \log \left|s_{E}\right|^{2}} .
$$

By a direct calculation, $\operatorname{Ric}\left(h_{\epsilon}\right) \geq k \omega_{1, \epsilon}$ in the current sense. $\tau$ has compact support and

$$
\lim _{\epsilon \rightarrow 0} \int_{M=\bar{M} \backslash D}|\tau|_{h_{\epsilon}, k \omega_{1, \epsilon}}^{2}\left(k \omega_{1, \epsilon}\right)^{n}=\int_{M}|\tau|_{h, k \omega}^{2}\left(k \omega_{1}\right)^{n}<\infty .
$$

So by the above corollary, there exists $\varsigma_{\epsilon}$ on $M$ such that

$$
\bar{\partial} \varsigma_{\epsilon}=\tau, \quad \int_{M}\left|\varsigma_{\epsilon}\right|_{h_{\epsilon}}^{2}\left(k \omega_{1, \epsilon}\right)^{n} \leq \int_{M}|\tau|_{h_{\epsilon}, k \omega_{1, \epsilon}}^{2}\left(k \omega_{1, \epsilon}\right)^{n}
$$

for each $\epsilon$. This also implies

$$
\int_{M}\left|\varsigma_{\epsilon}\right|_{h}^{2}\left(k \omega_{1}\right)^{n}<\infty
$$

Hence we can take a subsequence of $\varsigma_{\epsilon}$ converging weakly in $L^{2}\left(M,\left(k \omega_{1}\right)^{n}\right)$ to $\varsigma$ and

$$
\bar{\partial} \varsigma=\tau, \quad \int_{M}\left|\varsigma_{\epsilon}\right|_{h}^{2}\left(k \omega_{1}\right)^{n} \leq \int_{M}|\tau|_{h, k \omega_{1}}^{2}\left(k \omega_{1}\right)^{n}
$$

on $M$. The proof is complete after pushing $\varsigma$ to $M_{\text {reg }}$.

5.4. local separation of points. Recall that $\Phi: \bar{M} \rightarrow \Phi(\bar{M})$ is defined as in section 3. Naturally, $\Phi$ induce a map $\Phi: \mathcal{R} \rightarrow \Phi(\bar{M} \backslash D)$. If $s \in H^{0}\left(\mathcal{R}, k\left(K_{\bar{M}}+D\right)\right)$, then by Proposition 5.15, we know that $s$ is local bounded and local Lipschitz. So $s$ can be continuous extended to the limit space $M_{1}$. Furthermore, the map $\Phi_{1}:\left(\mathcal{R}, \omega_{1}\right) \rightarrow\left(\Phi(\bar{M} \backslash D), \omega_{F S}\right)$ defined by $\Phi$ can be continuously extend to $\Phi_{1}:\left(M_{1}, d_{1}\right) \rightarrow\left(\Phi(\bar{M} \backslash D), \omega_{F S}\right)$. This subsection is devoted to demonstrate that this map is injective. First we recall some notations and results which originate from [13].

Definition 5.21. We consider the following data $\left(p_{*}, O, U, J, g, L, h, A\right)$ satisfying

(1) $\left(p_{*}, O, U, J, g\right)$ is an open Kähler manifold with a complex structure $J$, a Riemannian metric $g$ and $a$ base point $p_{*} \in O \subset \subset U$ for an open set $O$.

(2) $L \rightarrow U$ is a hermitian line bundle equipped with a hermitian metric $h$ and $A$ is the connection induced by the hermitian metric $h$ on $L$, with its curvature $\Theta(A)=\omega$ which is a Kähler form of $g$.

The data $\left(p_{*}, O, U, J, g, L, h, A\right)$ is said to satisfy the $H$-condition if there exist a constant $C$ and a compactly supported smooth section $\sigma: U \rightarrow L$ such that 
(1) $H_{1}:\|\sigma\|_{L^{2}(U)} \leq(2 \pi)^{\frac{n}{2}}$,

(2) $H_{2}:\left|\sigma\left(p_{*}\right)\right|>\frac{3}{4}$,

(3) $H_{3}$ : for any smooth section $\tau$ of $L$ over $O$, we have

$$
\left|\tau\left(p_{*}\right)\right| \leq C\left(\|\bar{\partial} \tau\|_{L^{2 n+1}(O)}+\|\tau\|_{L^{2}(O)}\right),
$$

(4) $H_{4}:\|\bar{\partial} \sigma\|_{L^{2}(U)}<\min \left(\frac{1}{8 \sqrt{2} C}, 10^{-20}\right)$,

(5) $H_{5}:\|\bar{\partial} \sigma\|_{L^{2 n+1}(O)}<\frac{1}{8 C}$.

Fix any point $p$ in $M_{1},\left(M_{1}, p, k d_{1}\right)$ converges in pointed Gromov-Hausdorff topology to a tangent cone $C(Y)$ over the cross section when $k \rightarrow \infty$. We still use $p$ for the vertex of $C(Y)$. Let $Y_{\text {reg }}$ and $Y_{\text {sing }}$ be the regular part and singular part of $Y$ respectively. By [4], $Y_{\text {sing }}$ has Hausdorff dimension equal or less than $2 n-2$. $C\left(Y_{\text {reg }}\right) \backslash\{p\}$ has a natural complex structure induced from the Gromov-Hausdorff limit and the cone metric $g_{C}$ on $C(Y)$ is given by

$$
\omega_{C}=\frac{1}{2} \sqrt{-1} \partial \bar{\partial} r^{2}
$$

where $r$ is the distance function for any point $z \in C(Y) \backslash p$. According to Proposition [5.5, the singular set $\mathcal{S}$ of $M_{1}$ must be a locally analytic set by taking the limit of a divisor on $\bar{M}$. So we also have the following cut-off function on $Y$.

Proposition 5.22. For any $\epsilon>0$, there exists a cut-off function $\gamma$ on $Y$ such that

(1) $\gamma \in C^{\infty}\left(Y_{\text {reg }}\right)$ and $0 \leq \gamma \leq 1$,

(2) $\gamma$ is supported in the $\epsilon$ neighborhood of $Y_{\text {sing }}$,

(3) $\gamma=1$ on a neighborhood of $Y_{\text {sing, }}$,

(4) $\|\nabla \gamma\|_{L^{2}\left(Y, g_{C}\right)}<\epsilon$.

We consider the trivial line bundle $L_{C}$ on $C(Y)$ equipped the hermitian metric $h_{C}=e^{-|z|^{2}}$, where $|z|^{2}=r^{2}$. Then the curvature coincides with $\omega_{C}$. $A_{C}$ is denoted by the connection of $L_{C}$ with metric $h_{C}$.

Lemma 5.23. Let $p_{*} \in C\left(Y_{\text {reg }}\right) \backslash\{p\}$ such that $\frac{3}{4}<e^{-\left|p_{*}\right|^{2}}$. Then there exists $U \subset \subset C\left(Y_{\text {reg }}\right) \backslash\{p\}$ and an open neighborhood $O \subset \subset U$ of $p_{*}$ such that $\left(p_{*}, O, U, J_{C}, g_{C}, L_{C}, h_{C}, A_{C}\right)$ satisfies the $H$-condition.

From the construction in [13, $U$ is a product in $C\left(Y_{\text {reg }}\right) \backslash\{p\}$ i.e., there exists $U_{Y} \subset Y_{\text {reg }}$ such that $U=\left\{z=(y, r) \in C(Y) \mid y \in U_{Y}, r \in\left(r_{U}, R_{U}\right)\right\}$. For $m \in \mathbb{Z}^{+}$defined as [13] (P79), we define

$$
U(m)=\left\{z=(y, r) \in C(Y) \mid y \in U_{Y}, r \in\left(m^{-\frac{1}{2}} r_{U}, R_{U}\right)\right\} .
$$

For any integer $t$ and $1 \leq t \leq m, \mu_{t}: U \rightarrow U(m)$ is defined by $\mu_{t}(z)=t^{-\frac{1}{2}} z$. The following proposition is due to [13].

Proposition 5.24. Suppose $\left(p_{*}, O, U(m), J_{C}, g_{C}, L_{C}, h_{C}, A_{C}\right)$ constructed as in Lemma 5.23 satisfies the $H$-condition. If $\left(p_{*}, O, U(m), J, g, L, h, A\right)$ satisfies $H$-condition and there exists a small constant $\epsilon>0$ such that

$$
\left\|g-g_{C}\right\|_{C^{0}(U(m))}+\left\|J-J_{C}\right\|_{C^{0}(U(m))}<\epsilon
$$

Then we can find some $1 \leq t \leq m$ such that $\left(p_{*}, O, U, \mu_{t}^{*} J, \mu_{t}^{*}(t g), \mu_{t}^{*}\left(L^{t}\right), \mu_{t}^{*}\left(h^{t}\right), \mu_{t}^{*}\left(A^{t}\right)\right)$ satisfies $H$-condition. 
Fix any point $p$, we can assume that $\left(M_{1}, k_{v}^{\frac{1}{2}} d_{1}, p\right)$ converge to a tangent cone $C\left(Y_{p}\right)$ for some sequence $k_{v}$ in pointed Gromov-Hausdorff topology. For an open set $U \subset \subset C\left(Y_{\text {reg }}\right) \backslash\{p\}$, there is an embedding $\chi_{k_{v}}: U \rightarrow \mathcal{R}=M_{\text {reg }}$. Note that $\left.d_{1}\right|_{\mathcal{R}}=\omega_{1}$. The following Lemma follows from the convergence of $\left(M_{1}, k_{v}^{\frac{1}{2}} d_{1}, p\right)$.

Lemma 5.25. There exist $v$ and $\epsilon>0$ such that we can find an embedding $\chi_{k_{v}}$ which satisfies

(1) $\frac{1}{2}|z| \leq k_{v}^{\frac{1}{2}} d_{1}\left(p, \chi_{k_{v}}(z)\right) \leq 2|z|$,

(2) $\left\|\chi_{k_{v}}^{*}\left(k_{v} \omega_{1}\right)-\omega_{C}\right\|_{C^{0}(U)}+\left\|\chi_{k_{v}}^{*}\left(J_{\mathcal{R}}\right)-J_{C}\right\|_{C^{0}(U)}<\epsilon$.

Proposition 5.26. For any two distinct point $p$ and $q$ in $M_{1}$, we have

$$
\Phi_{1}(p) \neq \Phi_{1}(q) .
$$

Proof. Step 1: For any two distinct points $p$ and $q$, there exist $r$ and $R$ such that $p, q \in B_{d_{1}}\left(x_{1}, r\right) \subset$ $B_{d_{1}}\left(x_{1}, 2 r\right) \subset B_{d_{1}}\left(x_{1}, R\right)$. Suppose $C\left(Y_{p}\right)$ and $C\left(Y_{q}\right)$ are two tangent cones of $p$ and $q$ after rescaling $\left(M_{1}, d_{1}\right)$ at $p$ by $k_{v_{p}} \rightarrow \infty$ and at $q$ by $k_{v_{q}} \rightarrow \infty$. Then according to Lemma 5.23, we can construct two collection of data $\left(p_{*}, O_{p}, U_{p}\left(m_{p}\right), J_{p}, g_{p}, L_{p}, h_{p}, A_{p}\right)$ and $\left(q_{*}, O_{q}, U_{q}\left(m_{q}\right), J_{q}, g_{q}, L_{q}, h_{q}, A_{q}\right)$ which satisfy the $H$-condition, where $U_{p}\left(m_{p}\right) \subset C\left(Y_{p}\right)$ and $U_{q}\left(m_{q}\right) \subset C\left(Y_{q}\right)$. In addition, we can always assume that

(1) the constant $C$ appeared in the $H$-condition for $U_{p}\left(m_{p}\right)$ and $U_{q}\left(m_{q}\right)$ are the same,

(2) $k_{v_{p}}=k_{v_{q}}=k_{v_{p, q}}$,

(3) $r_{p_{*}}:=d_{C\left(Y_{p}\right)}\left(p, p_{*}\right)$ and $r_{q_{*}}:=d_{C\left(Y_{q}\right)}\left(q, q_{*}\right)$ are small enough which definite below.

Step 2: From Lemma 5.25, there exist $k_{v_{p, q}}$ such that $\chi_{p, k_{v, q}}: U_{p}\left(m_{p}\right) \rightarrow \mathcal{R}$ and $\chi_{q, k_{v_{p}, q}}: U_{q}\left(m_{q}\right) \rightarrow$ $\mathcal{R}$ satisfy the following:

(1) $\frac{1}{2}|z| \leq k_{v_{p, q}}^{\frac{1}{2}} d_{1}\left(p, \chi_{p}(z)\right) \leq 2|z|$,

(2) $\frac{1}{2}|z| \leq k_{v_{p, q}}^{\frac{1}{2}} d_{1}\left(q, \chi_{q}(z)\right) \leq 2|z|$,

(3) $\chi_{p}\left(U_{p}\left(m_{p}\right)\right) \cap \chi_{q}\left(U_{q}\left(m_{q}\right)\right)=\varnothing$,

(4) $\left\|\chi_{p}^{*}\left(k_{v_{p, q}} \omega_{1}\right)-\omega_{p}\right\|_{C^{0}\left(U_{p}\left(m_{p}\right)\right)}+\left\|\chi_{p}^{*}\left(J_{\mathcal{R}}\right)-J_{p}\right\|_{C^{0}\left(U_{p}\left(m_{p}\right)\right)}<\epsilon$,

(5) $\left\|\chi_{q}^{*}\left(k_{v_{p, q}} \omega_{1}\right)-\omega_{q}\right\|_{C^{0}\left(U_{q}\left(m_{q}\right)\right)}+\left\|\chi_{q}^{*}\left(J_{\mathcal{R}}\right)-J_{q}\right\|_{C^{0}\left(U_{q}\left(m_{q}\right)\right)}<\epsilon$.

where for the convenience, $\chi_{p, k_{v, q}}$ and $\chi_{q, k_{v, q}}$ are denoted by $\chi_{p}$ and $\chi_{q}$ respectively.

Step 3: By the Proposition 5.24 and sufficiently small $\epsilon$ in Step 2, there exists $1 \leq t_{p} \leq m_{p}$ such that $\left(p_{*}, O_{p}, U_{p}, \mu_{t_{p}}^{*} \chi_{p}^{*}\left(J_{\mathcal{R}}\right), \mu_{t_{p}}^{*} \chi_{p}^{*}\left(k_{v_{p, q}} \omega_{1}\right), \mu_{t_{p}}^{*} \chi_{p}^{*}\left(L_{p}^{t_{p}}\right), \mu_{t_{p}}^{*} \chi_{p}^{*}\left(h_{p}^{t_{p}}\right), \mu_{t_{p}}^{*} \chi_{p}^{*}\left(A_{p}^{t_{p}}\right)\right)$ satisfies the $H$-condition. Thus there is a compactly smooth section $\sigma_{p}$ such that $\sigma_{p}$ has properties $H_{1}, H_{2}, H_{4}$ and $H_{5}$. By the same argument, there exists $1 \leq t_{q} \leq m_{q}$ such that $\left(q_{*}, O_{q}, U_{q}, \mu_{t_{q}}^{*} \chi_{q}^{*}\left(J_{\mathcal{R}}\right), \mu_{t_{q}}^{*} \chi_{q}^{*}\left(k_{v_{p, q}} \omega_{1}\right), \mu_{t_{q}}^{*} \chi_{q}^{*}\left(L_{q}^{t_{q}}\right)\right.$, $\left.\mu_{t_{q}}^{*} \chi_{q}^{*}\left(h_{q}^{t_{q}}\right), \mu_{t_{q}}^{*} \chi_{q}^{*}\left(A_{q}^{t_{q}}\right)\right)$ satisfies the $H$-condition. Thus there is a compactly smooth section $\sigma_{q}$ such that $\sigma_{q}$ has properties $H_{1}, H_{2}, H_{4}$ and $H_{5}$.

Step 4: There is an embedding from $\left(\mu_{t_{p}}^{*} \chi_{p}^{*}\left(L_{p}^{t_{p}}\right), U_{p}\right)$ to $\left(k_{p}\left(K_{\bar{M}}+D\right), \mathcal{R}\right)$, where $k_{p}=t_{p} k_{v_{p, q}}$. So $\sigma_{p}$ can be viewed as a compactly smooth section of $k_{p}\left(K_{\bar{M}}+D\right)$. We now apply Proposition 5.20 to $\tau_{p}=\bar{\partial} \sigma_{p}$. Then there exists a $\left(k_{p}\left(K_{\bar{M}}+D\right)\right)$ valued section $\varsigma_{p}$ solving the $\bar{\partial}$ equation $\bar{\partial} \varsigma_{p}=\tau_{p}$ with

$$
\int_{\mathcal{R}}\left|\varsigma_{p}\right|^{2}\left(k_{p} \omega_{1}\right)^{n} \leq \int_{\mathcal{R}}\left|\tau_{p}\right|^{2}\left(k_{p} \omega_{1}\right)^{n} \leq \min \left(\frac{1}{8 \sqrt{2} C}, 10^{-20}\right) .
$$

Let $z_{p_{*}}=\chi_{p}\left(p_{*}\right)$, then from $H_{3}$ and $H_{5}$,

$$
\varsigma_{p}\left(z_{p_{*}}\right) \leq C\left(\left\|\bar{\partial} \varsigma_{p}\right\|_{L^{2 n+1}\left(O_{p}\right)}+\left\|\varsigma_{p}\right\|_{L^{2}\left(O_{p}\right)}\right) \leq \frac{1}{8 \sqrt{2} C}+\frac{1}{8 C} \leq \frac{1}{4} .
$$


Set $\sigma_{p}^{\prime}=\sigma_{p}-\varsigma_{p}$. Then $\sigma_{p}^{\prime}$ is a holomorphic section of $k_{p}\left(K_{\bar{M}}+D\right)$ over $\mathcal{R}$ and from Proposition 5.15 , $\sigma_{p}^{\prime}$ can be continuously extended to $M_{1}$. By the $H$-condition, we have the following relations:

(1) $\left|\sigma_{p}^{\prime}\left(z_{p_{*}}\right)\right|>\frac{1}{2}$

(2) $\left\|\sigma_{p}^{\prime}\right\|_{L^{2}\left(\mathcal{R}, k_{p} \omega_{1}, h_{p}^{k_{p}}\right)} \leq 2(2 \pi)^{\frac{n}{2}}$

(3) $\left\|\sigma_{p}^{\prime}\right\|_{L^{2}\left(\mathcal{R} \backslash U_{p}, k_{p} \omega_{1}, h_{p}^{k_{p}}\right)}=\left\|\varsigma_{p}\right\|_{L^{2}\left(\mathcal{R} \backslash U_{p}, k_{p} \omega_{1}, h_{p}^{k_{p}}\right)} \leq \min \left(\frac{1}{8 \sqrt{2} C}, 10^{-20}\right)$.

Then by Proposition 5.15 .

$$
\left|\sigma_{p}^{\prime}(p)\right| \geq\left|\sigma_{p}^{\prime}\left(z_{p_{*}}\right)\right|-\sup _{B_{d_{1}}\left(x_{1}, r\right)}\left|\nabla \sigma_{p}^{\prime}\right| k_{p}^{\frac{1}{2}} d_{1}\left(p, z_{p_{*}}\right) \geq \frac{2}{5}
$$

when $r_{p_{*}}$ is sufficiently small.

Now we restrict $\sigma_{p}^{\prime}$ on $U_{q}$. By $H_{3}$,

$$
\left|\sigma_{p}^{\prime}\left(z_{q_{*}}\right)\right| \leq C|| \sigma_{p}^{\prime} \|_{L^{2}\left(\mathcal{R} \backslash U_{p}, k_{p} \omega_{1}, h_{p}^{k_{p}}\right)} \leq C \min \left(\frac{1}{8 \sqrt{2} C}, 10^{-20}\right) .
$$

Similarly,

$$
\left|\sigma_{p}^{\prime}(q)\right| \leq\left|\sigma_{p}^{\prime}\left(z_{q_{*}}\right)\right|+\sup _{B_{d_{1}}\left(x_{1}, r\right)}\left|\nabla \sigma_{p}^{\prime}\right| k_{p}^{\frac{1}{2}} d_{1}\left(q, z_{q_{*}}\right) \leq 2 C \min \left(\frac{1}{8 \sqrt{2} C}, 10^{-20}\right),
$$

when $r_{p_{*}}$ is sufficiently small.

Step 5: By the same argument of Step 4, let $k_{q}=t_{q} k_{v_{p, q}}$, we construct a holomorphic section $\sigma_{q}^{\prime}$ such that

$$
\left|\sigma_{q}^{\prime}(q)\right| \geq \frac{2}{5}, \quad\left|\sigma_{q}^{\prime}(p)\right| \leq 2 C \min \left(\frac{1}{8 \sqrt{2} C}, 10^{-20}\right) .
$$

Step 6: Set $K=t_{q} k_{p}=t_{p} k_{q}$. Then $\left(\sigma_{p}^{\prime}\right)^{t_{q}}$ and $\left(\sigma_{q}^{\prime}\right)^{t_{p}}$ are holomorphic section of $K(K \bar{M}+D)$ which can be continuously extended to $M_{1}$. Modifying the constant $10^{-20}$ as small as enough, we have $\left|\left(\sigma_{p}^{\prime}\right)^{t_{q}}(p)\right|>>\left|\left(\sigma_{q}^{\prime}\right)^{t_{p}}(p)\right|$ and $\left|\left(\sigma_{q}^{\prime}\right)^{t_{p}}(q)\right|>>\left|\left(\sigma_{p}^{\prime}\right)^{t_{q}}(q)\right|$. Therefore, we conclude that $\Phi_{1}$ is injective.

5.5. Surjectivity of $\Phi_{1}$. In this subsection we will complete the proof of Theorem 1.8 . Let $u_{1}$ be the solution to the following equation in the current sense

$$
\left(\eta_{1}-\sqrt{-1} \partial \bar{\partial} \log \log ^{2}\left|s_{D}\right|^{2}+\sqrt{-1} \partial \bar{\partial} u_{1}\right)^{n}=e^{u_{1}} \frac{\Omega}{\left|s_{D}\right|^{2} \log ^{2}\left|s_{D}\right|^{2}} .
$$

Since $K_{\bar{M}}+D$ is big and semi-ample, there exists an effective divisor $E=\sum_{i} a_{i} E_{i}$ such that $K_{\bar{M}}+$ $D-\epsilon E>0$ for all sufficiently small $\epsilon>0$.

Let $p \in \operatorname{Supp} E \backslash D$ and $\pi: \widetilde{\bar{M}} \rightarrow \bar{M}$ be the blow up at $p$ with exceptional divisor $\pi^{-1}(p)=F$. Set $\widetilde{D}=\pi^{-1}(D)$ and $\widetilde{E}=\sum_{i} a_{i} \widetilde{E_{i}}$, where $\widetilde{E_{i}}=\overline{\pi^{-1}\left(E_{i}\right)-F} \cdot s_{\widetilde{E_{i}}}, s_{F}$ and $s_{\widetilde{D}}$ are denoted by the defining sections of line bundles $L_{\widetilde{E_{i}}}, L_{F}$ and $L_{\widetilde{D}}$ respectively. Let $\chi$ be fixed Kähler metric on $\widetilde{\bar{M}}$. We choose appropriate hermitian metrics $h_{\widetilde{E_{i}}}$ and $h_{F}$ such that

$$
\pi^{*} \eta_{1}+\delta \sqrt{-1} \partial \bar{\partial} \log \left|s_{F}\right|^{2}+\delta \sum_{i} a_{i} \sqrt{-1} \partial \bar{\partial} \log \left|s_{E_{i}}\right|^{2} \geq \mu \chi
$$




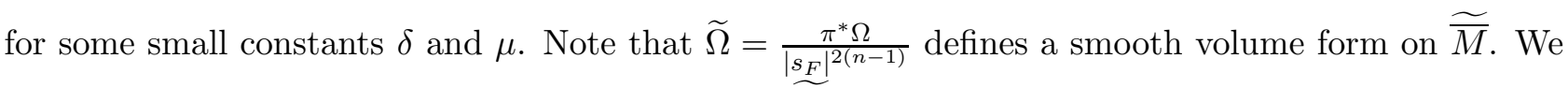
consider the following Monge-Ampère equation on $\widetilde{\bar{M}}$

$$
\left(\widetilde{\eta_{1}}+\epsilon \chi+\sqrt{-1} \partial \bar{\partial} \varphi_{\epsilon}\right)^{n}=e^{\varphi_{\epsilon}}\left(\epsilon^{2}+\left|s_{F}\right|^{2}\right)^{n-1} \frac{\widetilde{\Omega}}{\left|s_{\widetilde{D}}\right|_{h_{\widetilde{D}}}^{2} \log ^{2}\left|s_{\widetilde{D}}\right|_{h_{\tilde{D}}}^{2}}
$$

where $\widetilde{\eta}_{1}=\pi^{*} \eta_{1}-\sqrt{-1} \partial \bar{\partial} \log \log ^{2}\left|s_{\widetilde{D}}\right|_{h_{\tilde{D}}}^{2}$ and $h_{\widetilde{D}}=\pi^{*} h_{D}$. By Theorem 1 of [16], the equation has a unique smooth solution $\varphi_{\epsilon}$ for each $\epsilon$; moreover

$$
\widetilde{\omega_{\epsilon}}:=\widetilde{\eta_{1}}+\epsilon \chi+\sqrt{-1} \partial \bar{\partial} \varphi_{\epsilon}
$$

is a smooth complete Kähler metric on $\widetilde{\bar{M}} \backslash \widetilde{D}$.

Lemma 5.28. For any $\delta$ and $\epsilon$, there exist two constants $C(\delta)$ and $C$ independent of $\epsilon$ such that

$$
-C(\delta)+\delta \log \left|s_{F}\right|^{2}+\delta \sum_{i} a_{i} \log \left|s_{\widetilde{E_{i}}}\right|^{2} \leq \varphi_{\epsilon} \leq C+\log \log ^{2}\left|s_{\widetilde{D}}\right|_{h_{\widetilde{D}}}^{2} .
$$

Proof. For the upper bound, let

$$
V_{\epsilon}=\int\left(\epsilon^{2}+\left|s_{F}\right|^{2}\right)^{n-1} \frac{\widetilde{\Omega}}{\left|s_{\widetilde{D}}\right|_{h_{\tilde{D}}}^{2} \log ^{2}\left|s_{\widetilde{D}}\right|_{h_{\tilde{D}}}^{2}}
$$

so we have $V_{1} \geq V_{\epsilon} \geq V_{0}$. Hence $V_{\epsilon}$ is uniformly bounded. Denote $\left(\epsilon^{2}+\left|s_{F}\right|^{2}\right)^{n-1} \frac{\widetilde{\Omega}}{\left|s_{\widetilde{D}}\right|_{h}^{2} \log ^{2}\left|s_{\tilde{D}}\right|_{h_{\widetilde{D}}}^{2}}$ by $\widetilde{\Omega_{\epsilon}}$, then we have the following calculation

$$
\begin{aligned}
\frac{1}{V_{\epsilon}} \int \varphi_{\epsilon} \widetilde{\Omega_{\epsilon}} & =\frac{1}{V_{\epsilon}} \int \log \left(\frac{\widetilde{\omega}_{\epsilon}^{n}}{\widetilde{\Omega_{\epsilon}}}\right) \widetilde{\Omega_{\epsilon}} \leq \log \int \widetilde{\omega}_{\epsilon}^{n}-\log V_{\epsilon} \\
& =\log \int\left(\pi^{*} \eta+\epsilon \chi\right)^{n}-\log V_{\epsilon} \leq C,
\end{aligned}
$$

where the third equality bases on a Lemma ([16] P410). Since $\varphi_{\epsilon}-\log \log ^{2}\left|s_{\widetilde{D}}\right|_{h_{\tilde{D}}}^{2} \in P S H\left(\widetilde{\bar{M}}, \pi^{*} \eta+\epsilon \chi\right)$, the mean inequality implies

$$
\sup \varphi_{\epsilon} \leq C+\log \log ^{2}\left|s_{\widetilde{D}}\right|_{h_{\widetilde{D}}}^{2}
$$

For the lower bound, we set $\varphi_{\epsilon, \delta}=\varphi_{\epsilon}-\delta \log \left|s_{F}\right|^{2}-\delta \sum_{i} a_{i} \log \left|s_{E_{i}}\right|^{2}$ and denote $\left|s_{\widetilde{D}}\right|_{h_{\widetilde{D}}^{\delta}}^{2}=\left|s_{\widetilde{D}}\right|_{\delta}^{2}$, then the equation (5.27) is equivalent to

$$
\begin{aligned}
& \left(\eta_{1}^{\delta}+\delta \sqrt{-1} \partial \bar{\partial} \log \left|s_{F}\right|^{2}+\delta \sum_{i} a_{i} \sqrt{-1} \partial \bar{\partial} \log \left|s_{\widetilde{E_{i}}}\right|^{2}+\epsilon \chi+\sqrt{-1} \partial \bar{\partial} \varphi_{\epsilon, \delta}+\sqrt{-1} \partial \bar{\partial} \log \frac{\log ^{2}\left|s_{\widetilde{D}}\right|_{\delta}^{2}}{\log ^{2}\left|s_{\widetilde{D}}\right|^{2}}\right)^{n} \\
& =e^{\varphi_{\epsilon, \delta}+\log \frac{\log ^{2}\left|s_{\tilde{D}}\right|{ }_{\delta}^{2}}{\log ^{2}\left|s_{\widetilde{D}}\right|^{2}}} \cdot \prod_{i}\left|s_{\mathbb{E}_{i}}\right|^{2 a_{i} \delta} \cdot\left|s_{F}\right|^{2 \delta} \cdot\left(\epsilon^{2}+\left|s_{F}\right|^{2}\right)^{n-1} \cdot \frac{\widetilde{\Omega}^{\prime}}{\left|s_{\widetilde{D}}\right|_{\delta}^{2} \log ^{2}\left|s_{\widetilde{D}}\right|_{\delta}^{2}}
\end{aligned}
$$


where $\eta_{1}^{\delta}=\pi^{*} \eta_{1}-\sqrt{-1} \partial \bar{\partial} \log \log ^{2}\left|s_{\widetilde{D}}\right|_{\delta}^{2}$ satisfying $\eta_{1}^{\delta}+\delta \sqrt{-1} \partial \bar{\partial} \log \left|s_{F}\right|^{2}+\delta \sum_{i} a_{i} \sqrt{-1} \partial \bar{\partial} \log \left|s_{E_{i}}\right|^{2}>0$ and $\widetilde{\Omega}^{\prime}=\frac{\left|s_{\tilde{D}}\right|_{\delta}^{2}}{\left|s_{\widetilde{D}}\right|^{2}} \widetilde{\Omega}$. We introduce the following equation

$$
\left(\eta_{1}^{\delta}-\delta \sum_{i} \Theta_{\widetilde{E_{i}}}-\delta \Theta_{F}+\epsilon \chi+\sqrt{-1} \partial \bar{\partial} \psi_{\epsilon, \delta}\right)^{n}=e^{\psi_{\epsilon, \delta}} \cdot\left(\epsilon^{2}+\left|s_{F}\right|^{2}\right)^{n-1} \cdot \frac{\widetilde{\Omega}^{\prime}}{\left|s_{\widetilde{D}}\right|_{\delta}^{2} \log ^{2}\left|s_{\widetilde{D}}\right|_{\delta}^{2}}
$$

By the generalized maximum principle, there exists a sequence $\left\{x_{i}\right\}$ such that $\lim _{i \rightarrow \infty} \psi_{\epsilon, \delta}\left(x_{i}\right)=\inf \psi_{\epsilon, \delta}$ and $\overline{\lim }_{i \rightarrow \infty} \sqrt{-1} \partial \bar{\partial} \psi_{\epsilon, \delta}\left(x_{i}\right) \geq 0$. Then we have

$$
\inf \psi_{\epsilon, \delta} \geq(n-1) \log \frac{1}{\epsilon^{2}+\left|s_{F}\right|^{2}}+\log \frac{\left|s_{\widetilde{D}}\right|_{\delta}^{2} \log ^{2}\left|s_{\widetilde{D}}\right|_{\delta}^{2}\left(\eta_{1}^{\delta}-\delta \sum_{i} \Theta_{\widetilde{E_{i}}}-\delta \Theta_{F}+\epsilon \chi\right)^{n}}{\widetilde{\Omega}^{\prime}} \geq-C(\delta) .
$$

Set $H_{\epsilon, \delta}=\varphi_{\epsilon, \delta}-\psi_{\epsilon, \delta}$ and $v_{\epsilon}^{\delta}=\eta_{1}^{\delta}-\delta \sum_{i} \Theta_{\widetilde{E}_{i}}-\delta \Theta_{F}+\epsilon \chi$, then

$$
\begin{aligned}
& \log \frac{\left(v_{\epsilon}^{\delta}+\sqrt{-1} \partial \bar{\partial} \psi_{\epsilon, \delta}+\sqrt{-1} \partial \bar{\partial} H_{\epsilon, \delta}+\sqrt{-1} \partial \bar{\partial} \log \frac{\log ^{2}\left|s_{\widetilde{D}}\right|_{\delta}^{2}}{\log ^{2}\left|s_{\widetilde{D}}\right|^{2}}\right)^{n}}{\left(v_{\epsilon}^{\delta}+\sqrt{-1} \partial \bar{\partial} \psi_{\epsilon, \delta}\right)^{n}} \\
= & H_{\epsilon, \delta}+\log \frac{\log ^{2}\left|s_{\widetilde{D}}\right|_{\delta}^{2}}{\log ^{2}\left|s_{\widetilde{D}}\right|^{2}}+\delta \log \left|s_{F}\right|^{2}+\delta \sum_{i} a_{i} \log \left|s_{E_{i}}\right|^{2} .
\end{aligned}
$$

By the generalized maximum principle again

$$
\inf \left(H_{\epsilon, \delta}+\log \frac{\log ^{2}\left|s_{\widetilde{D}}\right|_{\delta}^{2}}{\log ^{2}\left|s_{\widetilde{D}}\right|^{2}}\right) \geq-C(\delta) .
$$

Note that $\log \frac{\log ^{2}\left|s_{\tilde{D}}\right|_{\delta}^{2}}{\log ^{2}\left|s_{\widetilde{D}}\right|^{2}}$ is a smooth function on $\widetilde{\bar{M}}$, so it can be bounded by $C(\delta)$. Moreover we get the lower bound of $\varphi_{\epsilon}$.

Lemma 5.29. There exists a constant $C$ independent of $\epsilon$ such that on $\widetilde{\bar{M}} \widetilde{D}$, we have

$$
\operatorname{Ric}\left(\widetilde{\omega_{\epsilon}}\right) \leq-\widetilde{\omega}_{\epsilon}+C \chi
$$

Proof. We observe some following consequences:

(1) $\pi^{*} \eta_{1} \leq C \chi$,

(2) Since $\widetilde{\Omega}$ is a smooth volume form, $\operatorname{Ric}(\widetilde{\Omega}) \leq C \chi$,

(3) $\Theta_{h_{\tilde{D}}} \geq-C \chi$

(4) $\sqrt{-1} \partial \bar{\partial} \log \left(\epsilon^{2}+\left|s_{F}\right|^{2}\right) \geq-C \chi$.

Thus by a simple calculation we get the Lemma.

Set $\chi^{\prime}=\chi-\sqrt{-1} \partial \bar{\partial} \log \log ^{2}\left|s_{\widetilde{D}}\right|^{2}$, then by a calculation we have

$$
\chi^{\prime}=\chi-2 \frac{\sqrt{-1} \partial \bar{\partial} \log \left|s_{\widetilde{D}}\right|_{\prime}^{2}}{\log \left|s_{\widetilde{D}}\right|_{\prime}^{2}}+2 \frac{\sqrt{-1} \partial \log \left|s_{\widetilde{D}}\right|_{\prime}^{2} \wedge \bar{\partial} \log \left|s_{\widetilde{D}}\right|_{\prime}^{2}}{\log ^{2}\left|s_{\widetilde{D}}\right|_{\prime}^{2}} .
$$

Take an appropriate hermitian metric $|\cdot|$, we can assume that

$$
\frac{1}{2} \chi \leq \frac{1}{2} \chi+2 \frac{\sqrt{-1} \partial \log \left|s_{\widetilde{D}}\right|_{\prime}^{2} \wedge \bar{\partial} \log \left|s_{\widetilde{D}}\right|_{\prime}^{2}}{\log ^{2}\left|s_{\widetilde{D}}\right|_{\prime}^{2}} \leq \chi^{\prime} \leq 2 \chi+2 \frac{\sqrt{-1} \partial \log \left|s_{\widetilde{D}}\right|_{\prime}^{2} \wedge \bar{\partial} \log \left|s_{\widetilde{D}}\right|_{\prime}^{2}}{\log ^{2}\left|s_{\widetilde{D}}\right|_{\prime}^{2}}
$$


So, by Lemma 5.29, we have

$$
\operatorname{Ric}\left(\widetilde{\omega_{\epsilon}}\right) \leq-\widetilde{\omega_{\epsilon}}+C \chi^{\prime}
$$

On the other hand, we can choose a sufficiently large $A^{\prime}$, a sufficiently small $\alpha$ and a hermitian metric $|\cdot|$ such that

$$
\begin{aligned}
& A^{\prime} \pi^{*} \eta_{1}-\Theta_{F}-\sum_{i} a_{i} \Theta_{\widetilde{E}_{i}}-A^{\prime} \sqrt{-1} \partial \bar{\partial} \log \log ^{2}\left|s_{\widetilde{D}}\right|^{2} \\
\geq & 3 \chi-2 A^{\prime} \frac{\sqrt{-1} \partial \bar{\partial} \log \left|s_{\widetilde{D}}\right|^{2}}{\log \left|s_{\widetilde{D}}\right|^{2}}+2 A^{\prime} \frac{\sqrt{-1} \partial \log \left|s_{\widetilde{D}}\right|^{2} \wedge \bar{\partial} \log \left|s_{\widetilde{D}}\right|^{2}}{\log ^{2}\left|s_{\widetilde{D}}\right|^{2}} \\
\geq & 2 \chi+2 A^{\prime} \frac{\sqrt{-1} \partial \log \left|s_{\widetilde{D}}\right|^{2} \wedge \bar{\partial} \log \left|s_{\widetilde{D}}\right|^{2}}{\log ^{2}\left|s_{\widetilde{D}}\right|^{2}} \geq \alpha \chi^{\prime} .
\end{aligned}
$$

From now on we always assume that the hermitian metric $|\cdot|$ on $L_{\widetilde{D}}$ satisfy $A^{\prime} \pi^{*} \eta_{1}-\Theta_{F}-\sum_{i} a_{i} \Theta_{\widetilde{E_{i}}}-$ $A^{\prime} \sqrt{-1} \partial \bar{\partial} \log \log ^{2}\left|s_{\widetilde{D}}\right|^{2} \geq \alpha \chi^{\prime}$.

Lemma 5.30. There exist $C$ and $\lambda$ independent of $\epsilon$ such that

$$
\widetilde{\omega_{\epsilon}} \leq \frac{C\left(\log ^{2}\left|s_{\widetilde{D}}\right|^{2}\right)^{C}}{\left|s_{\widetilde{D}}\right|^{2 \lambda} \cdot\left|s_{F}\right|^{2 \lambda^{2}} \cdot \prod_{i}\left|s_{E_{i}}\right|^{2 \lambda^{2}}} \chi^{\prime} .
$$

Proof. By Yau's Schwarz Lemma [25] and Lemma 5.29, we have

There is a fact that is

$$
\triangle_{\widetilde{\omega_{\epsilon}}} \log t r_{\chi^{\prime}} \widetilde{\omega}_{\epsilon} \geq-C t r_{\widetilde{\omega_{\epsilon}}} \chi^{\prime}-\frac{C}{t r_{\chi^{\prime}} \widetilde{\omega}_{\epsilon}}
$$

$$
\triangle_{\widetilde{\omega_{\epsilon}}} \varphi_{\epsilon} \leq n-\operatorname{tr}_{\widetilde{\omega_{\epsilon}}}\left(\pi^{*} \eta_{1}-\sqrt{-1} \partial \bar{\partial} \log \log ^{2}\left|s_{\widetilde{D}}\right|^{2}\right) .
$$

Let $H=\log \left(\left|s_{\widetilde{D}}\right|^{2 A} \cdot\left|s_{F}\right|^{2 A^{2}} \cdot \prod_{i}\left|s_{\widetilde{E}_{i}}\right|^{2 A^{2}} \cdot t r_{\chi^{\prime}} \widetilde{\omega}_{\epsilon}\right)-A^{2} A^{\prime} \varphi_{\epsilon}$, where $A^{\prime}$ is chosen as above and $A$ is defined below. Then on $\widetilde{\bar{M}} \backslash(\widetilde{D} \cup F \cup \operatorname{Supp} E)$, we have

$\triangle_{\widetilde{\omega_{\epsilon}}} H \geq-C t r_{\widetilde{\omega_{\epsilon}}} \chi^{\prime}-\frac{C}{\operatorname{tr}_{\chi^{\prime}} \widetilde{\omega}_{\epsilon}}-A^{2} A^{\prime} n+A \operatorname{tr}_{\widetilde{\omega_{\epsilon}}}\left(A A^{\prime} \pi^{*} \eta_{1}-A \Theta_{F}-A \sum_{i} a_{i} \Theta_{\widetilde{E_{i}}}-\Theta_{\widetilde{D}}-A A^{\prime} \sqrt{-1} \partial \bar{\partial} \log \log ^{2}\left|s_{\widetilde{D}}\right|^{2}\right)$.

When $A$ is sufficiently large we observed that

$$
\operatorname{Atr}_{\widetilde{\omega_{\epsilon}}}\left(A\left(A^{\prime} \pi^{*} \eta_{1}-\Theta_{F}-\sum_{i} a_{i} \Theta_{\widetilde{E_{i}}}-A^{\prime} \sqrt{-1} \partial \bar{\partial} \log \log ^{2}\left|s_{\widetilde{D}}\right|^{2}\right)-\Theta_{\widetilde{D}}\right) \geq(C+1) \chi^{\prime} .
$$

Therefore

$$
\triangle_{\widetilde{\omega_{\epsilon}}} H \geq t r_{\widetilde{\omega_{\epsilon}}} \chi^{\prime}-\frac{C}{t r_{\chi^{\prime}} \widetilde{\omega}_{\epsilon}}-A^{2} A^{\prime} n
$$

By the generalized maximum principle, there exists a sequence $\left\{x_{i}\right\}$ such that $\lim _{i \rightarrow \infty} H\left(x_{i}\right)=\sup H$ and $\overline{\lim }_{i \rightarrow \infty} \sqrt{-1} \partial \bar{\partial} H\left(x_{i}\right) \leq 0$. Thus,

$$
\varlimsup_{i \rightarrow \infty} t r_{\chi^{\prime}} \widetilde{\omega}_{\epsilon} \cdot\left(t r_{\widetilde{\omega_{\epsilon}}} \chi^{\prime}-A^{2} A^{\prime} n\right)\left(x_{i}\right) \leq C .
$$

Since

$$
\widetilde{\omega}_{\epsilon}^{n}=e^{\varphi_{\epsilon}} \frac{\widetilde{\Omega}}{\left|s_{\widetilde{D}}\right|^{2} \log ^{2}\left|s_{\widetilde{D}}\right|^{2}} \leq C \log ^{2}\left|s_{\widetilde{D}}\right|^{2}\left(\chi^{\prime}\right)^{n},
$$


then we have

$$
\frac{1}{\log ^{2}\left|s_{\widetilde{D}}\right|^{2}}\left(\operatorname{tr}_{\chi^{\prime}} \widetilde{\omega}_{\epsilon}\right)^{\frac{1}{n-1}} \leq C \operatorname{tr}_{\widetilde{\omega_{\epsilon}}} \chi^{\prime}
$$

Furthermore,

If

$$
\varlimsup_{i \rightarrow \infty} t r_{\chi^{\prime}} \widetilde{\omega}_{\epsilon} \cdot\left(\frac{1}{C \log ^{2}\left|s_{\widetilde{D}}\right|^{2}}\left(\operatorname{tr}_{\chi^{\prime}} \widetilde{\omega}_{\epsilon}\right)^{\frac{1}{n-1}}-A^{2} A^{\prime} n\right)\left(x_{i}\right) \leq C .
$$

$$
\varlimsup_{i \rightarrow \infty}\left(t r_{\chi^{\prime}} \widetilde{\omega}_{\epsilon}\right)^{\frac{1}{n-1}}\left(x_{i}\right) \leq \varlimsup_{i \rightarrow \infty} 2 A^{2} A^{\prime} n C \log ^{2}\left|s_{\widetilde{D}}\right|^{2}\left(x_{i}\right)
$$

then

Otherwise

$$
\varlimsup_{i \rightarrow \infty}\left(\operatorname{tr}_{\chi^{\prime}} \widetilde{\omega}_{\epsilon}\right)\left(x_{i}\right) \leq \varlimsup_{i \rightarrow \infty}\left(2 A^{2} A^{\prime} n C\right)^{n-1}\left(\log ^{2}\left|s_{\widetilde{D}}\right|^{2}\right)^{n-1}\left(x_{i}\right) .
$$

$$
\varlimsup_{i \rightarrow \infty}\left(t r_{\chi^{\prime}} \widetilde{\omega}_{\epsilon}\right)^{\frac{1}{n-1}}\left(x_{i}\right) \geq \varlimsup_{i \rightarrow \infty} 2 A^{2} A^{\prime} n C \log ^{2}\left|s_{\widetilde{D}}\right|^{2}\left(x_{i}\right) .
$$

From (5.31) we know

$$
\varlimsup_{i \rightarrow \infty} A^{2} A^{\prime} n t r_{\chi^{\prime}} \widetilde{\omega}_{\epsilon}\left(x_{i}\right) \leq C .
$$

In general we have

$$
\varlimsup_{i \rightarrow \infty}\left(t r_{\chi^{\prime}} \widetilde{\omega}_{\epsilon}\right)\left(x_{i}\right) \leq \varlimsup_{i \rightarrow \infty} C\left(\log ^{2}\left|s_{\widetilde{D}}\right|^{2}\right)^{C}\left(x_{i}\right)
$$

By the definition of $H$ and Lemma 5.28 we have

$$
\begin{aligned}
H(x) \leq \overline{\lim }_{i \rightarrow \infty}\left(\log \left(\left|s_{\widetilde{D}}\right|^{2 A} \cdot\left|s_{F}\right|^{2 A^{2}} \cdot \prod_{i}\left|s_{\widetilde{E_{i}}}\right|^{2 A^{2}} \cdot C\left(\log ^{2}\left|s_{\widetilde{D}}\right|^{2}\right)^{C}\right)\right. \\
\left.+A^{2} A^{\prime} C(\delta)-A^{2} A^{\prime} \delta \log \left|s_{F}\right|^{2}-A^{2} A^{\prime} \delta \sum_{i} a_{i} \log \left|s_{\widetilde{E_{i}}}\right|^{2}\right)\left(x_{i}\right) \leq C
\end{aligned}
$$

when choosing $A>>A^{\prime}$ and sufficiently small $\delta$. So we get this Lemma from the upper bound of $\varphi_{\epsilon}$.

Let $B$ be a disk in $\bar{M} \backslash D$ centered at $p$. Denote $f_{1}, f_{2}, \cdots, f_{N}$ by the defining functions of divisors $\widetilde{E_{1}}, \widetilde{E_{2}}, \cdots, \widetilde{E_{N}}$ on $\widetilde{B}=\pi^{-1}(B)$. From Lemma 5.30, we obtain the following corollary.

Corollary 5.32. There exist $C$ and $\lambda$ independent of $\epsilon$ such that

$$
\left.\left(\operatorname{tr}_{\chi^{\prime}} \widetilde{\omega}_{\epsilon}\right)\right|_{\partial \widetilde{B}} \leq\left.\frac{C}{\prod_{i}\left|f_{i}\right|^{2 \lambda^{2}}}\right|_{\partial \widetilde{B}} .
$$

Let $\hat{\chi}$ be the pull back of the Euclidean metric $\sqrt{-1} \sum_{j} d z_{j} \wedge d \overline{z_{j}}$ on $B$. Then $\hat{\chi}$ is a smooth closed nonnegative $(1,1)$ form and is a Kähler metric on $\widetilde{B} \backslash F$. The following Lemma is due to Song [21].

Lemma 5.33. There exist a constant $C>0$, a sufficiently small $\epsilon_{0}>0$ and a smooth hermitian metric $h_{F}^{\prime}$ on $L_{F}$ such that on $\widetilde{B}$

$$
C^{-1} \hat{\chi} \leq \chi^{\prime} \leq C \frac{\hat{\chi}}{\left|s_{F}\right|_{h_{F}^{\prime}}^{2}}
$$

and

$$
\pi^{*} \eta_{1}-\sqrt{-1} \partial \bar{\partial} \log \log ^{2}\left|s_{\widetilde{D}}\right|^{2}-\epsilon_{0} \Theta_{h_{F}^{\prime}}>C^{-1} \chi^{\prime}
$$


Lemma 5.34. There exist $0<\beta<1, C>0$ and $\Lambda>0$ independent of $\epsilon$ such that

$$
\widetilde{\omega}_{\epsilon} \leq \frac{C}{\left|s_{F}\right|_{h_{F}^{\prime}}^{2(1-\beta)} \cdot \prod_{i}\left|f_{i}\right|^{2 \Lambda}} \chi^{\prime}, \text { in } \widetilde{B} \text {. }
$$

Moreover, we have

$$
\pi^{*} \omega_{1} \leq \frac{C}{\left|s_{F}\right|_{h_{F}^{\prime}}^{2(1-\beta)} \cdot \prod_{i}\left|f_{i}\right|^{2 \Lambda}} \chi^{\prime}, \quad \text { in } \widetilde{B},
$$

where $\omega_{1}=\eta_{1}-\sqrt{-1} \partial \bar{\partial} \log \log ^{2}\left|s_{D}\right|^{2}+\sqrt{-1} \partial \bar{\partial} u_{1}$.

Proof. Let $H=\log \left(\left|s_{F}\right|_{h_{F}^{\prime}}^{2(1+r)} \cdot \prod_{i}\left|f_{i}\right|^{2 \lambda^{2}} \cdot t r_{\hat{\chi}} \widetilde{\omega_{\epsilon}}\right)-A \varphi_{\epsilon}$ for some sufficiently large $A$ and sufficiently small $r$. There are some facts on $\widetilde{B} \backslash(F \cup \operatorname{Supp} E)$ :

(1) $\triangle_{\widetilde{\omega_{\epsilon}}} \log \left|s_{F}\right|_{h_{F}^{\prime}}^{2}=-\operatorname{tr}_{\widetilde{\omega_{\epsilon}}} \Theta_{h_{F}^{\prime}}$,

(2) $\triangle_{\widetilde{\omega_{\epsilon}}} \log \prod_{i}\left|f_{i}\right|^{2 \lambda^{2}}=0$,

(3) $\triangle_{\widetilde{\omega_{\epsilon}}} \varphi_{\epsilon}=n-t r_{\widetilde{\omega_{\epsilon}}}\left(\pi^{*} \eta_{1}-\sqrt{-1} \partial \bar{\partial} \log \log ^{2}\left|s_{\widetilde{D}}\right|^{2}\right)-\epsilon r_{\widetilde{\omega_{\epsilon}}} \chi$,

(4) $\triangle_{\widetilde{\omega_{\epsilon}}} \log \operatorname{tr}_{\hat{\chi}} \widetilde{\omega_{\epsilon}} \geq-\frac{\operatorname{tr}_{\hat{\chi}}\left(\operatorname{Ric}\left(\widetilde{\omega_{\epsilon}}\right)\right)}{\operatorname{tr}_{\hat{\chi}} \widetilde{\omega_{\epsilon}}} \geq 1-\frac{C}{\left|s_{F}\right|_{h_{F}^{\prime}}^{2} \operatorname{tr}_{\hat{\chi}} \widetilde{\omega}_{\epsilon}}$.

Thus, on $\widetilde{B} \backslash(F \cup \operatorname{Supp} E)$, we have

$$
\begin{aligned}
\triangle_{\widetilde{\omega_{\epsilon}}} H & \geq 1-\frac{C}{\left|s_{F}\right|_{h_{F}^{\prime}}^{2} \operatorname{tr}_{\hat{\chi}} \widetilde{\omega_{\epsilon}}}-A n-(r+1) \operatorname{tr}_{\widetilde{\omega_{\epsilon}}} \Theta_{h_{F}^{\prime}}+A t r_{\widetilde{\omega_{\epsilon}}}\left(\pi^{*} \eta_{1}-\sqrt{-1} \partial \bar{\partial} \log \log ^{2}\left|s_{\widetilde{D}}\right|^{2}\right) \\
& \geq \operatorname{ctr}_{\widetilde{\omega_{\epsilon}}} \chi^{\prime}-\frac{C}{\left|s_{F}\right|_{h_{F}^{\prime}}^{2} \operatorname{tr}_{\tilde{\chi}} \widetilde{\omega_{\epsilon}}}-C,
\end{aligned}
$$

where the last inequality bases on Lemma 5.33 and choosing sufficiently large $A$ and small $r$. By Yau's Schwarz Lemma 25],

$$
\triangle_{\widetilde{\omega_{\epsilon}}} \log t r_{\chi^{\prime}} \widetilde{\omega}_{\epsilon} \geq-C_{1} t r_{\widetilde{\omega_{\epsilon}}} \chi^{\prime}-\frac{C_{1}}{t r_{\chi^{\prime}} \widetilde{\omega}_{\epsilon}}
$$

Let $G=H+\frac{c}{2 C_{1}} \log \left(\prod_{i}\left|f_{i}\right|^{2 \lambda^{2}+2} \cdot t r_{\chi^{\prime}} \widetilde{\omega}_{\epsilon}\right)$. Then

$$
\begin{aligned}
\triangle_{\widetilde{\omega_{\epsilon}}} G & \geq c t r_{\widetilde{\omega_{\epsilon}}} \chi^{\prime}-\frac{C}{|s|_{h_{F}^{\prime}}^{2} t r_{\hat{\chi}} \widetilde{\omega_{\epsilon}}}-C-\frac{c}{2} t r_{\widetilde{\omega_{\epsilon}}} \chi^{\prime}-\frac{c}{2 t r_{\chi^{\prime}} \widetilde{\omega_{\epsilon}}} \\
& \geq \frac{c}{2} t r_{\widetilde{\omega_{\epsilon}}} \chi^{\prime}-\bar{C}-\frac{C}{\left|s_{F}\right|_{h_{F}^{\prime}}^{2} t r_{\hat{\chi}} \widetilde{\omega_{\epsilon}}} .
\end{aligned}
$$

For a fixed sufficiently large $\lambda>0$, there exists a constant $C>0$ such that

$$
\sup _{\partial \widetilde{B}} G \leq C
$$

from the estimate in Corollary 5.32 and Lemma 5.28 .

So we can assume that

$$
\sup _{\widetilde{B}} G=G\left(p_{\max }\right)
$$

for some $p_{\max } \in \widetilde{B} \backslash(F \cup \operatorname{Supp} E)$. Then at $p_{\max }$, we have

$$
\left|s_{F}\right|_{h_{F}^{\prime}}^{2} \operatorname{tr}_{\hat{\chi}} \widetilde{\omega}_{\epsilon}\left(c t r_{\widetilde{\omega_{\epsilon}}} \chi^{\prime}-2 \bar{C}\right)\left(p_{\max }\right) \leq C .
$$


Note that

$$
\frac{1}{\log ^{2}\left|s_{\widetilde{D}}\right|^{2}}\left(\operatorname{tr}_{\chi} \widetilde{\omega}_{\epsilon}\right)^{\frac{1}{n-1}} \leq C \operatorname{tr}_{\widetilde{\omega_{\epsilon}}} \chi^{\prime}
$$

Then according to the boundedness of $\frac{1}{\log ^{2}\left|s_{\widetilde{D}}\right|^{2}}$ in $\widetilde{B}$, we get

$$
\left|s_{F}\right|_{h_{F}^{\prime}}^{2} \operatorname{tr}_{\hat{\chi}} \widetilde{\omega}_{\epsilon}\left(C^{\prime}\left(\operatorname{tr}_{\chi^{\prime}} \widetilde{\omega}_{\epsilon}\right)^{\frac{1}{n-1}}-2 \bar{C}\right)\left(p_{\max }\right) \leq C .
$$

If $\left(\operatorname{tr}_{\chi^{\prime}} \widetilde{\omega}_{\epsilon}\right)^{\frac{1}{n-1}}\left(p_{\max }\right) \leq \frac{3 \bar{C}}{C^{\prime}}$, then $G$ is bounded from above by a uniform constant.

Otherwise $\left(\operatorname{tr}_{\chi^{\prime}} \widetilde{\omega}_{\epsilon}\right)^{\frac{1}{n-1}}\left(p_{\max }\right) \geq \frac{3 \bar{C}}{C^{\prime}}$, i.e., $\bar{C} \leq \frac{C^{\prime}}{3}\left(\operatorname{tr}_{\chi^{\prime}} \widetilde{\omega}_{\epsilon}\right)^{\frac{1}{n-1}}\left(p_{\max }\right)$. Then by equation (5.35) we get

$$
\left|s_{F}\right|_{h_{F}^{\prime}}^{2} \cdot \operatorname{tr}_{\hat{\chi}} \widetilde{\omega}_{\epsilon} \cdot \frac{C^{\prime}}{3}\left(\operatorname{tr}_{\chi^{\prime}} \widetilde{\omega}_{\epsilon}\right)^{\frac{1}{n-1}}\left(p_{\max }\right) \leq C,
$$

i.e.

$$
\log \left|s_{F}\right|_{h_{F}^{\prime}}^{2}+\log t r_{\hat{\chi}} \widetilde{\omega}_{\epsilon}+\frac{1}{n-1} \log t r_{\chi^{\prime}} \widetilde{\omega}_{\epsilon}\left(p_{\max }\right) \leq C .
$$

According to the definition of $G$, Lemma 5.28 and Lemma 5.30 we have $G \leq C$ when we choose large $C_{1}$.

In sum, in all cases, we have $G \leq C$. Then

$$
\left|s_{F}\right|_{h_{F}^{\prime}}^{2(1+r)} \cdot \prod_{i}\left|f_{i}\right|^{2 \lambda^{2}+\frac{c}{2 C_{1}}\left(2 \lambda^{2}+2\right)} \cdot \operatorname{tr}_{\hat{\chi}} \widetilde{\omega}_{\epsilon} \cdot\left(t r_{\chi^{\prime}} \widetilde{\omega}_{\epsilon}\right)^{\frac{c}{2 C_{1}}} \leq C
$$

Note that $\operatorname{tr}_{\hat{\chi}} \widetilde{\omega}_{\epsilon} \geq C^{-1} \operatorname{tr}_{\chi^{\prime}} \widetilde{\omega}_{\epsilon}$, then we observe

$$
\left(t r_{\chi^{\prime}} \widetilde{\omega}_{\epsilon}\right)^{1+\frac{c}{2 C_{1}}} \leq \frac{C}{\left|s_{F}\right|_{h_{F}^{\prime}}^{2(1+r)} \cdot \prod_{i}\left|f_{i}\right|^{2 \lambda^{2}+\frac{c}{2 C_{1}}\left(2 \lambda^{2}+2\right)}} .
$$

If we choose $r=\frac{c}{10 C_{1}}$, then $1-\beta=\frac{1+r}{1+\frac{c}{2 c_{1}}}$ for some $\beta \in(0,1)$. Furthermore, there exists a constant $\Lambda>0$ such that

$$
\widetilde{\omega_{\epsilon}} \leq \frac{C}{\left|s_{F}\right|_{h_{F}^{\prime}}^{2(1-\beta)} \cdot \prod_{i}\left|f_{i}\right|^{2 \Lambda}} \chi^{\prime} .
$$

From now on we turn to the Gromov-Hausdorff convergence. Recall

$$
\overline{D_{1}}:=\left\{x \in M_{1} \mid \text { there exists } x_{i} \in \bar{D} \text { such that } x_{i} \stackrel{d_{G H}}{\longrightarrow} x\right\},
$$

where $\bar{D}$ is a divisor such that $D \cup \mathcal{S}_{\bar{M}} \subset \bar{D}$. By the Proposition [5.5. $\left(M_{1} \backslash \overline{D_{1}}, \overline{\omega_{1}}\right)$ isometric to $\left(\bar{M} \backslash \bar{D}, \omega_{1}\right)$.

Lemma 5.36. $\Phi_{1}: M_{1} \backslash \overline{D_{1}} \rightarrow \Phi(\bar{M} \backslash \bar{D})$ is bijective.

Proof. Note that $\left(M_{1} \backslash \overline{D_{1}}\right) \subset \mathcal{R}=M_{\text {reg }}$ and $\left.\Phi\right|_{M_{\text {reg }}}$ is biholomorphic, so $\Phi_{1}$ is bijective.

Lemma 5.37. $\Phi_{1}: \overline{D_{1}} \rightarrow \Phi(\bar{D} \backslash D)$ is surjective.

Proof. For any $x^{\prime} \in \bar{D} \backslash D$, there exists a curve $\gamma:[0,1] \rightarrow \bar{M} \backslash D$ with $\gamma(0)=x^{\prime}$ and $\gamma((0,1]) \subset \bar{M} \backslash \bar{D}$ such that $\int_{0}^{1}|\dot{\gamma}|_{\omega_{1}} d t<\infty$ by Lemma 5.34. The curve $\gamma(t)$ gives a curve $\bar{\gamma}(t)$ for $0<t \leq 1$ through an isometry from $\left(\bar{M} \backslash \bar{D}, \omega_{1}\right)$ to $\left(M_{1} \backslash \overline{D_{1}}, \overline{\omega_{1}}\right)$. Hence there is a limit $x^{\prime \prime}=\lim _{t \rightarrow 0} \bar{\gamma}(t)$ in $M_{1}$. Then

$$
\Phi_{1}\left(x^{\prime \prime}\right)=\lim _{t \rightarrow 0} \Phi_{1}(\bar{\gamma}(t))=\lim _{t \rightarrow 0} \Phi(\gamma(t))=\Phi\left(x^{\prime}\right) .
$$


Therefore, $\Phi_{1}$ is surjective.

\section{REFERENCES}

[1] M. Anderson, Convergence and rigidity of manifolds under Ricci curvature bounds, Invent. Math. 102 (1990), 429445.

[2] R. J. Berman and H. Guenancia, Kähler-Einstein merics on stable varieties and log canonical pairs, Geom. Funct. Anal. 24 (2014), 1683C1730.

[3] S. Boucksom, A. Broustet, and G. Pacienza, Uniruledness of stable base loci of adjoint linear systems via Mori theory, Math. Z. 275 (2013), 499C507.

[4] J. Cheeger, Degeneration of Riemannian Metrics under Ricci Curvature Bounds, Lezioni Fermiane. Scuola Normale Superiore, Pisa (2001).

[5] J. Cheeger and T. H. Colding, On the structure of spaces with Ricci curvature bounded below II, J. Diff. Geom. 54 (2000), 13-35.

[6] X.X. Chen, S. Donaldson, and S. Sun, Kähler-Einstein metric on Fano manifolds, I: approximation of metrics with cone singularities, J. Amer. Math. Soc. 28 (2014), 183-197.

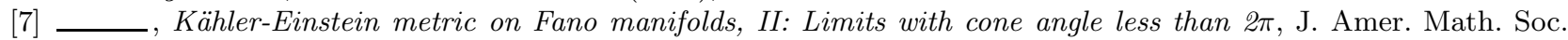
28 (2015), no. 1, 199-234.

[8] _ Kähler-Einstein metric on Fano manifolds, III: Limits as cone angle approaches $2 \pi$ and completion of the main proof, J. Amer. Math. Soc. 28 (2015), no. 1, 235-278.

[9] S. Y. Cheng and S. T. Yau, On the existence of a complete Kähler metric on non-compact complex manifolds and the regularity of Fefferman's equation, Communication on Pure and Applied Mathematics XXXIII (1980), 507-544.

[10] T. H. Colding, Ricci curvature and volume convergece, Annal. of Math. 145 (1997), 477-501.

[11] T. H. Colding and A. Naber, Sharp Hölder continuity of tangent cones for spaces with a lower Ricci curvature bound and applications, Annal. of Math. 176 (2012), 1173-1229.

[12] J. P. Demailly, Analytic methods in algebraic geometry.

[13] S. Donaldson and S. Sun, Gromov-Hausdorff limits of Käjler manifolds and algebraic geometry, Acta. math. 213 (2014), 63-106.

[14] D. Gilbarg and N. S. Trudinger, Elliptic partial differential equations of second order, Springer, 1983.

[15] Y. Kawamata, K. Matsuda, and K. Matsuki, An Introduction to the Minimal Model Problem, Advanced Studies in Pure Mathematics 10 (1987), 283C360.

[16] R. Kobayashi, Kähler-Einstein metric on an open algebraic manifold, Osaka1. Math. 21 (1984), 399-418.

[17] J. Kollar and F. Mori, Birational geometry of algebraic varieties, Cambridge Tracts in Mathematics, vol. 134, Cambridge University Press, 1998.

[18] G. La Nave and G. Tian, A continuity method to construct canonical metrics, arXiv:1410.3157.

[19] G. La Nave, G. Tian, and Z. L. Zhang, Bounding diameter of singular Kähler metric, arXiv:1503.03159v1.

[20] X.C. Rong and Y.G. Zhang, Continuity of extremal transitions and flops for Calabi-Yau manifolds, J. Diff. Geom. 89 (2011), 233-269.

[21] J. Song, Riemannian geometry of Kähler-Einstein currents, arXiv:1404.0445.

[22] G. Tian, K-stability and Kähler-Einstein metrics, Commun. Pure Appl. Math LXVIII (2015), 1085-1156.

[23] G. Tian and S. T. Yau, Existence of Kähler-Einstein mertics on complete Kähler manifolds and their applications to algebraic geometry, Adv. Ser. Math. Phys. 1, 1 (1987), 574-628.

[24] G. Tian and Z. L. Zhang, Convergence of Kähler Ricci flow on lower dimension algebraic manifold of general type arXiv: 1501.01038.

[25] S. T. Yau, A general Schwarz lemma for Kähler manifolds, Amer. J. of Math. 100 (1978), 197-208.

YAN LI

Beijing International Center for Mathematical Research,

PEKING UNIVERSITY,

100871 BEIJING, CHINA

E-mail address: liyandota@hotmail.com 